\title{
Connectivity in the Commercial Internet ${ }^{1}$
}

\author{
Jacques Crémer $^{2} \quad$ Patrick Rey $^{3} \quad$ Jean Tirole $^{4}$
}

May 1999

\footnotetext{
${ }^{1}$ The authors have advised GTE on the Internet aspects of the WorldCom-MCI merger, and are grateful to GTE for letting them use work done on that case for academic purposes. We should stress however that the views presented in this paper are our own, and need not reflect GTE's. We are grateful to Scott Flick, Scott Marcus and Jim Venit, for helpful discussions on the technology and economics of the Internet. This paper was presented at the conference on "Competition and Innovation in the Personal Computer Industry", San Diego, 24 April 1999. We thank the sponsors of this conference for support and the participants for comment.

${ }^{2}$ IDEI and GREMAQ (CNRS UMR 5604), Toulouse.

${ }^{3}$ IDEI and GREMAQ (CNRS UMR 5604), Toulouse.

${ }^{4}$ IDEI and GREMAQ (CNRS UMR 5604), Toulouse, CERAS (CNRS UMR 2036), and MIT.
} 


\begin{abstract}
In this paper, we study the "backbone market" in the Internet. The first part of the paper discusses the structure of the Internet and shows that there does exist a separate backbone market. The second part uses an extension of the Katz-Shapiro network model to analyze the strategies that would be used by a firm that dominates the backbone market. We first show that in this market when two backbones interconnect the larger one prefers a lower quality interconnection than the smaller one. We then analyze the profitability of a "targeted degradation" strategy where a larger backbone would lower the quality of interconnection to its smaller rivals in turn. Finally, we analyze the incentives that clients would have to multihome in order to avoid the degradation of the quality of interconnection, and show that the main qualitative results are not affected.
\end{abstract}




\section{Introduction}

The Internet has entered a critically important period of transition from government ownership to commercial exploitation. Until recently, the Internet community was largely one of engineers working cooperatively to take the Internet off the ground, and the largest part of the initial network, the NSFNET, was privatized as late as 1995. In the commercial era that is just beginning, financial stakes are huge, and the Internet is turning into a fascinating commercial battleground.

While players in the industry are trying to design business models and contractual arrangements for the new environment, economic analysis has yet to produce guidance for strategy and competition policy. In fact, little is known about the "industrial organization of the Internet". The early work on the economics of the Internet focused on the use of smart market auctions and peak-load pricing to allocate scarce transmission capacity among competitive end users. ${ }^{1}$ While this work is clearly relevant, in this paper we turn our attention on the strategic behavior of firms and on the role of antitrust policy in the commercial Internet environment.

We will address only a small subset of the myriad fascinating questions related to these issues, focusing on the issue of connectivity. The Internet is a system of interconnected computer networks. In this industry characterized by strong network externalities, end users, consumers and businesses, seek ubiquitous connectivity and purchase connectivity from Internet service providers (ISPs). Internet backbone providers (IBPs) provide high bandwidth long-haul transmission, routing and interconnection to these ISPs and to their own vertically integrated ISPs and Web-hosting services. But is there a proper "backbone market", or should one consider IBPs as Internet service providers like all others and thus part of a broader market? And, if an IBP market indeed exists, what strategies can a player with substantial market power employ to enhance dominance?

These questions stood at the heart of the joint investigation in 1998 by the US Department of Justice and the European Commission of the proposed merger between WorldCom and MCI, which each owned one of the four largest Internet backbones. Antitrust authorities feared that the merged entity would have incentives to degrade the quality of its interconnection with the rest of the Internet, to introduce proprietary standards, or to impose tough interconnection agreements on other backbones. As a result of the investigation, the parties were required to divest about half of their Internet

\footnotetext{
${ }^{1}$ See, e.g., MacKie-Mason \& Varian $(1995 a, 1995 b)$ Gupta, Stahl \& Whinston (1994) and Shenker, Clark, Estrin \& Herzog (1996).
} 
assets before they were allowed to merge. ${ }^{2}$

The paper is organized as follows. Section 2 presents a brief overview of the industry, and explains the role of backbones. Section 3 performs a market definition and concentration analysis, thereby providing further details about demand substitution, supply response and potential entry.

Our formal analysis builds on Katz and Shapiro's classic 1985 "model of sponsorship" in industries with network externalities. Each backbone has an installed base and otherwise competes for unattached customers. The benefit derived by a customer from joining a backbone is an increasing function of the size of his or her backbone and, in a complementary fashion, of the size of the other backbones and of the quality of interconnection with the other backbones. This quality of interconnection is a strategic variable, and because "it takes two to tango", the quality of interconnection is governed by the preferences of the backbone which values interconnection the least.

The results obtained throughout the paper all rest on the comparison between two impacts, on the backbone contemplating degradation, of a change in the quality of interconnection with another backbone. First, when connectivity between the two networks is degraded, both backbones face a demand reduction, as their customers' access to each others deteriorates. Second, a degradation of connectivity creates a quality differentiation between the two networks. The larger backbone, which relies relatively less on access to the other backbone's customers, gains a competitive advantage, and competition between the two backbones is softened. However, when other backbones are present, a similar quality differentiation effect also handicaps both backbones relative to the other ones.

Section 4 analyzes the competition between two backbones of different sizes, and shows that the larger backbone has suboptimal incentives to maintain connectivity. Thanks to its larger installed base, the dominant backbone also acquires dominance in the market for unattached customers when connectivity is not perfect. The poorer the interconnection and the stronger the network externality, the more dominant is this backbone, which, not surprisingly, is less eager to interconnect than its rival. Degradation is more likely, the larger the difference in installed bases. Section 5 discusses modeling assumptions and the robustness of the conclusions.

Section 6 extends the analysis to different backbones configurations. First, it shows that if there are four equal-sized backbones, none has an incentive to degrade interconnection. Intuitively, a backbone that degrades the quality of

\footnotetext{
${ }^{2}$ MCI sold its Internet assets (connecting 1, 300 ISPs, 60, 000 business customers and 250, 000 consumers) to Cable \& Wireless. It also agreed to transfer 1, 000 employees to Cable \& Wireless and agreed not to woo back any customer for a period of two years. In March 1999, Cable \& Wireless sued MCI WorldCom, alleging violations of the agreement.
} 
its interconnection with an equal-size backbone does not gain a competitive advantage over this backbone, and its quality relative to that of the other two backbones deteriorates.

Second, we assume that two of these backbones merge and show that the new backbone, with its 50\% market share, still has no incentives to degrade simultaneously its two connections with the smaller backbones. However, we show that it can be optimal for the dominant backbone to degrade interconnection with one of the smaller backbones, that is to employ a "targeted degradation strategy".

Section 7 extends the analysis to allow for the possibility that customers connect to several backbones (multihome) and analyzes whether multihoming impairs a dominance-enhancing degradation strategy. It first looks at the impact of pre-existing multihoming by a fraction of the installed base. Keeping the difference in sizes of installed bases constant, it shows that preexisting multihoming is conducive to degradation by a dominant backbone. Intuitively, pre-existing multihoming lowers the pain of degradation (the reduction of demand), as well as, in the case of targeted degradation, the reduction in the competitive advantage enjoyed by the dominant backbone over the nontargeted backbone; and it does not affect quality differentiation between the targeting and targeted backbones. Interestingly, in the targeted degradation scenario, the dominant backbones prefers to target the small backbone with whom it has the most extensive customer overlap.

Second, we allow new customers and singlehoming installed-base customers to attempt to circumvent the degradation policy by connecting to several backbones. We show that the conclusions are for the most part unchanged. Intuitively, customers choose to multihome only if the price charged by the second backbone to which they connect is smaller than the value that they attach to the new connections that it provides. But in equilibrium, the price charged by each network reflects the value of the customer pool it uniquely gives access to. Second-homing to the dominant network brings high connectivity benefits but is expensive; second-homing to a smaller network is cheaper but brings low connectivity benefits. Thus, multihoming may not occur despite a degraded interconnection. Finally, we show that if multihoming occurs in reaction to a degraded interface, the smaller backbone's installed base is more likely to second-home to the dominant backbone than the dominant backbones's installed base to second-home to the smaller backbone. 


\section{Structure of the Internet}

\subsection{End users and network externalities}

End users include residential and business users, who have access to the Internet either through dial up (over the phone line using modems) or through dedicated access; and web sites, which provide a wide variety of free or feebased content as well as offerings of services (E-commerce,...).

As its name indicates, the Internet is defined by the fact that it enables connectivity. From its inception, it has been developed to enable communications between networks, and in its present state its most important feature is the ability for dial-up customers, Web sites hosts and dedicated access customers to exchange traffic across the entire system of interconnected networks.

This connectivity has been achieved first through the widespread adoption of the TCP and IP protocols, which support transmission of packets, irrespective of the type of data that they carry: text, video, voice, etc. The standardization of protocols would have been of no consequence without the build-up of interfaces between networks, first at the Network Access Points, and subsequently at private interconnects. The use of these interfaces has in turn been made possible by the development of a variety of contractual agreements between end-users and suppliers of Internet services, and between these suppliers.

It is important to note that the Internet's basic architecture was chosen and implemented by the US government, and especially the Department of Defense and the NSF, with much technical assistance from the academic community. The NSF stopped managing the Internet and funding the NSFNET on 30 April 1995, although it continues funding research designed to improve its functioning. ${ }^{3}$ The Internet has become the largest example of a deregulated communications network. However, it largely functions thanks to the institutions, standards and protocols that were chosen when the NSF was managing it, which in the long term will probably progressively lose their importance as technology evolves.

The benefits of connectivity arise because there are very strong network externalities. Network externalities exist when the value for a customer of

\footnotetext{
${ }^{3}$ The NSF funded the construction by MCI of the very high speed backbone Network Service (vBNS), which operates at OC-12 (622 Mbps) and interconnects through ATM switches a number of NSF-sponsored research institutions. More recently, it has provided impetus for Internet II (Abilene) for academia, and the NGI for government (for more on the Internet activities of the NSF since privatization, see Marcus (forthcoming, chapter 14)).
} 
belonging to a network increases with the number of customers in this network. ${ }^{4}$ In the case of the Internet, each customer profits in many direct and indirect ways from the presence of other customers. For instance, individuals derive direct benefits from the fact that friends and acquaintances are able to receive and send E-mail. A firm derives direct benefits from the fact that a government agency builds a Web site where it can find the texts of regulations that affect its business. A new customer who connects to the Internet yields indirect benefits to existing customers, by increasing the incentives of government agencies, non-profit organizations and businesses to open new Internet sites. Consumers, whether individuals or organizations, can benefit fully from these network externalities only if connectivity is assured.

\subsection{Providers of connectivity}

Between end users can be found a host of intermediaries. Some intermediaries (for instance, search engines, portals, "infomediaries") provide users with guidance as to where to connect, what to buy and so forth. Other intermediaries provide transmission services: Internet Service Providers (ISPs) and Internet Backbone Providers (IBPs)

Internet backbone providers (IBPs) transmit data over large regions of the world using long-haul fiber-optic cables. They pick up the traffic generated by ISPs as well as that of their own customers and carry it over long distances, connecting to each other and exchanging data at multiple points under the so-called "peering agreements" (see below). The IBPs also have the most sophisticated routing tables ${ }^{5}$ of all Internet players.

As we already observed, the Internet is a network of interconnected networks. Indeed, one of the main appeals of the Internet is its current almost ubiquitous connectivity: From almost any point (URL address) in the network can be sent messages to almost any other point. One may wonder how a network of 7,000 ISPs (4,500 in the US) and 5 to 50 IBPs (depending on the exact definition of $\operatorname{IBPs}^{6}$ ) can offer such ubiquitous connectivity.

The answer to this question stems from the fact that the Internet has a basically hierarchical structure. As illustrated by figure 1, IBPs sit on top of the hierarchy, customers lie at the bottom, and ISPs (to which can be added regional networks) stand in between. This may seem surprising

\footnotetext{
${ }^{4}$ See, e.g., Rohlfs (1974), Katz \& Shapiro (1985), Farrell \& Saloner (1985), Tirole (1988, chapter 10) and the Journal of Industrial Economics Symposium on Compatibility (1992).

${ }^{5}$ At each node of the Internet, the routing table stores the instructions that the "router" uses to forward incoming messages to another node.

${ }^{6}$ The five big IBPs are Cable \& Wireless (who owns the Internet assets that belonged to MCI), Sprint, MCI WorldCom, GTE and AT\&T.
} 


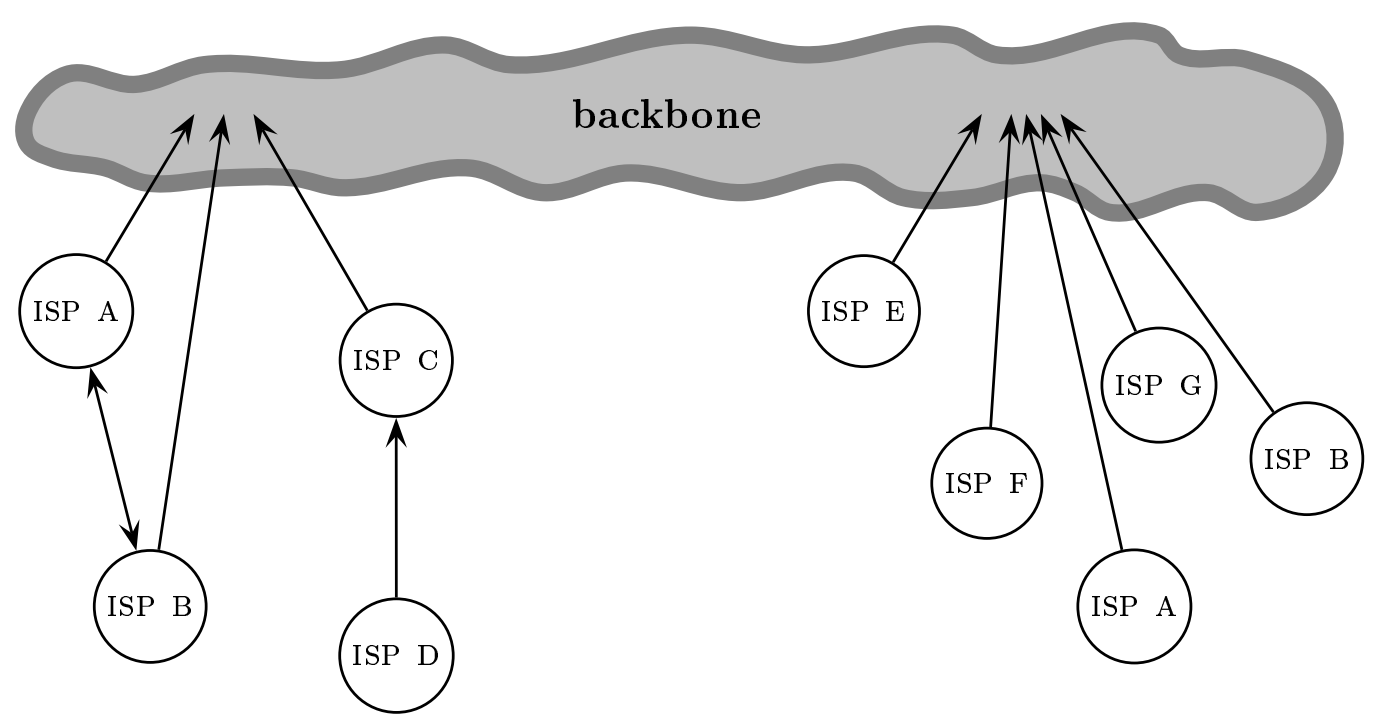

Figure 1: The configuration of the Internet.

since the Internet has been built to allow for very flexible organization and routing where the path of packets is optimized in real time depending on the loci of congestion in the network. However, a hierarchical structure offers several benefits. It facilitates routing by reducing the complexity of routing tables and by limiting the opportunities of gaming the system. A hierarchical structure also facilitates interconnection agreements by limiting the number of interconnection facilities, by clarifying responsibilities for immediacy, and by making it more transparent what installed base one is getting access to through a bilateral contract. Furthermore, and as we discuss in section 3, the hierarchical structure can accommodate certain types of non-hierarchical relationships while preserving its basic nature.

IBPs "peer" with each other. In so doing, they accept to route all traffic that is destined to their own customers, the customers of their customers, and so on. ${ }^{7}$ Peering used to occur at public peering points, NAPs (Network Access Points) or MAEs (Metropolitan Access Exchanges), where networks could exchange traffic. The slow expansion of the capacity at these points while Internet traffic grows at a tremendous rate has led IBPs to turn to private peering, that is to exchanging traffic pairwise at a number of bilateral

\footnotetext{
${ }^{7}$ Notice that peering arrangements are different from transit agreements where a party accepts to carry traffic for another one to a third party. Peering commits each provider to accept data destined to its customers, to the customers of its customers and so on, while transit commits the provider to also carry data destined for third parties.
} 
interfaces. IBPs impose a number of conditions to accept each other as peers: number and location of points of interface, ${ }^{8}$ national high-speed network, 24 hour-per-day network operation center, etc. Currently, peering arrangements are of the bill-and-keep type; that is, each peer terminates without charge the traffic originating with other peers. This feature is probably a leftover of the transition process, and one may wonder whether IBPs will keep running their two-way interconnection arrangements through bill-and-keep.

IBPs do not make money directly from their peering relationships. To recover their huge investments in infrastructure, they charge their customers, who in turn charge their own customers. Charges are related to the capacity of the link between the network and its customer, but can also depend on usage. Thus, the Internet can be seen as a pyramid, in which monies are collected at the bottom and passed through to the top of the hierarchy.

To be certain, the organization of the Internet is not purely hierarchical. For example, it may make sense for two ISPs, such as ISPs A and B in figure 1, who are in the same city, to exchange traffic directly (engage in "secondary peering") rather than let their mutual traffic move up and then down the hierarchy. Such sideway interconnections, however, do not contradict the fact that the Internet has a fundamentally hierarchical nature.

\subsection{Threats to connectivity}

Connectivity requires cooperation among firms that are otherwise competitors. They must reach bilateral agreements on the locations and capacities of interfaces, and on the financial terms through which they exchange traffic. All the major firms that provide Internet services must reach multilateral agreements on the protocols and standards that enable the exchange of traffic. Two trends will jeopardize this cooperation in the near future. First, as the operation of the Internet has been turned over to the private sector, and as a growing part of Internet service is provided by profit maximizing firms, conflicts of interest will become more pronounced; one cannot rely on the generalized goodwill that characterized the "Internet community" in the 1980s and early 1990s to ensure the future of the Internet. One of the

\footnotetext{
${ }^{8}$ One motivation for this is to make sure that an IBP will not suffer asymmetrically from "shortest exit routing". Suppose that most of the customers of IBP \#1 are located in San Francisco while the customers of IBP \#2 are evenly located in Boston and San Francisco. When a customer of IBP \#1 in San Francisco sends a message to a customer of IBP \#2 in Boston, IBP \#1 gets rid of the message as quickly as possible by transmiting it to IBP \#2 near San Francisco. The message is therefore carried mostly by the receiver's network. Conversely, IBP \#2 hands the return message (say, a web page download) to IBP \#1 as soon as possible, but cannot do so if the two IBPs do not interconnect near Boston.
} 
goals of this paper is to analyze the incentives large Internet players have to interconnect.

Second, "interconnection" involves different levels of quality. An obvious dimension of quality is delay. Ignoring delays occurring at the end user's location (e.g., web site), delays in the network occur both in the basic transport function (through the speed of transmission) and at switches (routers). These delays are managed at different levels on the Internet. In particular, if congestion builds up, the TCP protocol allows the routers to ask the senders of messages to slow down the rate at which data is sent. This limits the sizes of the queues that build up at the routers. ${ }^{9}$ Notice that these delays can occur both inside networks or at their interface. Current interconnection agreements are based on a "best effort" model; parties make a vague promise that they will do their best to limit delays on their own part of the exchanged traffic. While current delays are perfectly acceptable for services such as E-mail or Internet fax that do not require "immediacy", a number of new services require bounded delay. The development of real-time services such as Internet telephony, ${ }^{10}$ interactive teaching or video-conferencing (a surgeon in a teaching hospital giving real-time advice on an operation in a rural hospital) requires very low and very uniform delays between sender and receiver; new protocols will be needed to allow the development of these applications, and networks will need to cooperate in order to offer premium services at reasonable prices.

The development of premium services thus calls for new protocols sitting on top of the existing protocols, that will enable prioritizing messages, verifying delays, billing, etc., as well as for the design of innovative two-way access arrangements that induce players to offer these premium services. In contrast, "premium services connectivity" will be lost if some large Internet operators develop proprietary standards and offer such services on a limited basis (between their customers), hoping that the proprietary offering will create a competitive advantage.

Cooperation on bounded-delay services is only one of many of the challenging industrial organization issues in the Internet. Consider the development of multicast real-time services. A football game or a concert in a

\footnotetext{
${ }^{9}$ The traffic is sometimes very variable in the short run (it is said to be bursty); then it can increase rapidly enough that routers run out of buffer space to store queued packets before senders slow down. Then, some packets are discarded.

${ }^{10}$ Internet telephony, as its name indicates, refers to telephony over the Internet; unlike for a traditional phone call, for which a circuit is opened and dedicated to a single conversation for the whole length of the call, messages, like for other Internet services, are decomposed into tiny data packets, that may or may not take the same route, and are reassembled at termination.
} 
country may be simultaneously transmitted over the Internet to millions of viewers all over the world. In the current system, when a sender sends a packet to multiple receivers, the packet is replicated at the source and one copy is sent to each receiver. This obviously puts unnecessary strain on the Internet; for example a single copy could be forwarded to a router near a city and be replicated only at this router for the Internauts in that city who have signaled they wanted to join the group of viewers. The standardization of multicast routing requires cooperation among the players. ${ }^{11}$

Connectivity can in general be achieved in three ways: regulation, private negotiation among networks, and alternative methods, such as the customer's affiliation to multiple networks. These methods of achieving connectivity place the burden on different parties: the government in the case of regulation, the suppliers of service in the case of private negotiations, and the customers in the other cases.

Regulation of access has been the traditional way of guaranteeing interconnection for voice telephony, and this policy has been reaffirmed in the United States, by the Telecommunications Act of February 1996 and by the FCC, in the European Union, and indeed almost everywhere ${ }^{12}$ in the world. There is, however, a pronounced global trend toward reducing regulation and introducing enhanced competition in the telecommunications industry, and the 1996 US Telecommunications Act reaffirmed US policy that the Internet remain "unfettered by Federal or State regulation". It is therefore the competition authorities who must ensure, for example, that the benefits of connectivity not be jettisoned with the emergence of a dominant player who would take advantage of the network externalities to 'balkanize' the Internet and enhance its dominance.

\subsection{Customer loyalty}

Because the commercial Internet is recent and still in flux, we have limited knowledge of consumer behavior. Obviously, customers are highly heterogenous. Dial-up users, web sites, dedicated access customers and ISPs (as customers of other ISPs or IBPs) have different assessments of the pricequality trade-off, and furthermore, each category of customers exhibits high heterogeneity. Some customers, such as banks, value quality highly and are

\footnotetext{
${ }^{11}$ For more details on multicast services, see Shenker et al. (1996).

${ }^{12}$ One notable exception is New Zealand, which experimented with unregulated negotiations for interconnection between a dominant operator and smaller operators; this solution has not operated smoothly, however, even though it was scrutinized under articles 36 and 27 of the competition law and even though the threat of re-regulation may also have put some pressure on the dominant operator.
} 
not very price sensitive, since a loss of connectivity has dire consequences for them. On the other hand, Fortune 500 corporate headquarters may not care so much about small delays experienced by visitors of their web sites who consult their annual report. Some customers (such as universities) may be very price sensitive.

There is also a wide heterogeneity in switching costs. Like in any other industry, switching costs may be psychological (including a limited ability to rethink and alter at each point of time all contractual relationships one is engaged in), technical or contractual.

One determinant of the ease with which a customer can switch suppliers is the portability of IP addresses. For example, most ISPs obtain their address space from their connectivity supplier as part of their service contract, while the largest ones usually have their own address spaces. This hierarchical structure makes much sense from a routing perspective, but it creates substantial switching costs for those who do not have their own address space; similarly, a dedicated access customer may need to reconfigure its computers and renumber workstations following a switch. Based on high observed churning rates, physical switching costs for dial-up customers (who lose their E-mail addresses when they change ISPs) seem less substantial, although a proper econometric study should be conducted to assess the impact of poaching offers customer dissatisfaction on churn.

As in other industries, switching costs may also stem from contractual provisions. Dial-up customers, web-hosting customers, dedicated access customers and ISPs are often engaged in short-, medium- or long-term contracts with their supplier of connectivity (often a year to a couple of years for webhosting and dedicated-access customers; similarly, AOL is under a five-year long-term contract with WorldCom). There are several possible rationales for such long-term contracts: recovery of costs incurred by the supplier (connection, collocation, set-up service costs); planning of the supplier's network capacity and of the capacity and location of interfaces with other suppliers; price discrimination; ${ }^{13}$ as well as strategic behavior in a market with strong network externalities. The impact of long-term contracts and penalties for breach also differs widely among customers. For example, a web-hosting customer who signs a two-year contract specifying a base volume, may face a large switching cost if its volume stagnates or grows slowly, but only a small switching cost if its volume explodes (since he avoids paying the penalty for breach when reorienting most of the new demand to a new supplier).

Another interesting determinant of customer loyalty in the Internet is information. While price differentials are readily observable (at least upon

\footnotetext{
${ }^{13}$ See Fudenberg \& Tirole (1997).
} 
request), quality differentials may not be. ${ }^{14}$ A customer, say an ISP connecting to an IBP, experiencing poor connectivity from this IBP may not know whether the IBP or another IBP to whom the customer is contemplating switching, is responsible for the poor transmission quality.

\section{Market definition and concentration}

Despite its many flaws, the process of defining a market is a useful one. It obviously helps competition authorities to screen cases and reduces the transaction costs of antitrust enforcement; but, equally important, it serves as a preliminary exercise toward a fuller and more satisfactory study of "competitive effects". From the point of view of this paper, we sketch a market definition to bring out some further features of the Internet environment. We ask whether there is such a thing as a "backbone market". ${ }^{15}$

\subsection{Reminders about market definition}

The process of defining a relevant market is described in the Department of Justice 1992 Merger Guidelines for the US and the 1997 Commission Notice on the Definition of the Relevant Market for the European Union. The exercise of market power by a firm or group of firms acting collusively is constrained by consumer responses, as some consumers stop purchasing the product(s) sold by the firms in the group, and by supply responses, which include changes in production by firms outside the group, reconditioning and recycling of existing goods, and entry into the industry.

Only the former - the demand substitution effects - are considered in the definition of the market. Supply substitution is introduced when computing the market participants' market shares. Because distinguishing between supply and demand factors is not always easy from an economic perspective, ${ }^{16}$

\footnotetext{
${ }^{14}$ Boardwatch Magazine, though, publishes the Boardwatch Backbone Performance Index. This index uses three million measurements taken from a variety of locations over a 30 day period. Despite their imperfections (see Richard (1999)), such indices do convey information about relative performance.

${ }^{15}$ The issue of the existence of a backbone market was highly contentious in the 1998 WorldCom-MCI merger. The parties argued that there is no such thing as a backbone market and offered to include all ISPs in the definition of the relevant market. The European Commission and the Department of Justice thought otherwise.

${ }^{16}$ A supply can sometimes merely amount to a negative demand. For example, when an electricity customer reacts to an increase in the price of electricity by installing its own generating facilities, his reaction can be viewed either as a demand substitution or as a supply response. As a consequence of this ambiguity, the theory of pricing under market power insists that the relevant demand curve facing a firm with market power is
} 
and because we are mostly interested in a description of the industry, we will depart slightly from the legal definition and draw a broader picture by including supply responses into the market definition. ${ }^{17}$

Market definition in both the US and Europe rests on the hypothetical monopolist test. For example, the US Merger Guidelines state that

"A market is defined as a product or group of products and a geographic area in which it is produced or sold such that a hypothetical profit-maximizing firm, not subject to price regulation, that was the only present and future producer or seller of those products in that area likely would impose at least a "small but significant and nontransitory" increase in price, assuming the terms of sale of all other products are held constant. A relevant market is a group of products and a geographic area that is no bigger than necessary to satisfy this test."

The "small but significant" price increase is usually interpreted as a $5 \%$ (US) or 5 to $10 \%$ (Europe) increase, but there is flexibility in the choice of this magnitude.

Thus, consider a group of firms (which we will refer to as the hypothetical monopolist) and assume that these firms jointly raise their prices by 5 to $10 \%$; if this increase leads to an increase in the joint profit of the firms, their goods constitute a market. To fix ideas, assume that the firms in the group sell an homogenous good at a uniform price $p$. The quantities that we need to estimate can then easi1y be derived from elementary monopoly theory. Let $D(p)$ be the demand function for the services of the hypothetical monopolist, and let $c$ be the marginal cost of production. Ignoring fixed costs, the profit of the hypothetical monopolist is

$$
\Pi(p)=(p \Leftrightarrow c) D(p) .
$$

A small increase in price is profitable if

$$
\eta<\frac{p}{p \Leftrightarrow c},
$$

the residual demand curve.

${ }^{17}$ In Europe, "supply side substitutability may also be taken into account when defining markets in those situations in which its effects are equivalent to those of demand substitution in terms of effectiveness and immediacy. This requires that suppliers be able to switch production to the relevant products and market them in the short term without incurring significant additional costs or risks in response to small and permanent changes in relative prices. When these conditions are met, the additional production that is put on the market will have a disciplinary effect on the competitive behavior of the companies involved. Such an impact in terms of effectiveness and immediacy is equivalent to the demand substitution effect" (Commission Notice). Potential competition from new entrants however is not taken into account when defining markets. 
where $\eta=\Leftrightarrow p D^{\prime}(p) / D(p)$ is the price elasticity of demand. This elasticity is equal to the percentage decrease in the quantity demanded induced by a one percent increase in price. If we assume that the mark-up $(p \Leftrightarrow c) / p$ is equal to $50 \%$, the hypothetical monopolist test will be satisfied if the elasticity of demand for Internet backbone services is less than 2, that is if a $10 \%$ price increase in the price of backbone services induces a decrease in demand smaller than 20\%. Note that the strength of the test depends (as it should) on the competitiveness of the initial environment. Indeed, profits necessarily increase if the firms initially engage in cutthroat competition (price just above or below marginal cost). ${ }^{18}$

As is well known, applying the hypothetical monopolist test is not straightforward. Nonlinear pricing as well as third-degree price discrimination raise the issue of what "price" is being increased. For example, large customers bargain for special deals. Large ISPs such as AOL, with its 16 million subscribers, may let IBPs compete for the privilege to be able to give access to their large installed bases. They thus may be able to pay lower prices than other customers. The multiplicity of prices however does not affect the logic of market definition. The question, in the context of Internet connectivity, is: Would a large ISP be able to obtain the same deal (or a deal within a 5 to 10 percent margin), were the largest IBPs to merge and form an hypothetical monopolist, as it is able to get in today's competitive environment?

Another and related issue with the application of the hypothetical monopolist test is that the choice of the level of price increase is not innocent. Suppose that the industry serves two types of customers. One class of customers has an outside opportunity and will switch if prices go up by $3 \%$. The other class is captive. In such a world, a $5 \%$ price increase is unprofitable if the elastic class is big enough, while a much larger price increase raises profit. [Merger guidelines in both the US and Europe recognize the difficulties posed by discrimination and allow for the consideration of "submarkets".]

Last, the impact of the price increase on profits is hard to assess in the absence of reliable cost and demand data. ${ }^{19}$

\footnotetext{
${ }^{18}$ Conversely, if firms initially collude to maintain prices close to the monopoly level, a price increase will necessarily lead to a reduction in profits.

${ }^{19} \mathrm{Also}$, while it considers a nontransitory price increase, the hypothetical monopolist test is in essence static in that it does not reflect dynamic pricing considerations such as introductory pricing, learning effects, or the building of installed bases - which is particularly important in network industries.
} 


\subsection{End-users demand substitution}

We now consider a group of firms consisting of the largest backbone service providers, ${ }^{20}$ which we will simply refer to as "the backbone", and ask whether this group would pass the hypothetical monopoly test. That is, we assume that all firms operating the backbone increase their prices by $10 \%$, so that all direct customers face an increase in their charges of $10 \%$ for all the traffic that they send through the backbone. ${ }^{21}$ We will refer to the backbone's direct access customers as ISPs, but we should keep in mind that those direct customers also include web-site hosts, universities and dedicated access business customers connected directly to the backbone.

This price increase generates a reduction in the demand for backbone services, through two effects: First, to the extent that ISPs pass through this price increase to the retail price, retail customers may reduce their demand for Internet services. This is the retail-level or end-users demand substitution. Second, ISPs may try to circumvent the price increase by establishing direct connections to each other. These circumventing strategies represent wholesale-level substitution and can involve bilateral arrangements (transit, secondary peering) or more ambitious multilateral arrangements. We argue here that retail-level substitution is unlikely to exert much pressure on the backbone, and discuss wholesale-level substitution in more detail in the next subsection.

In the absence of any wholesale-level substitution, an increase in the price of backbone services induces an increase in the prices charged by the ISPs to their dial-up and other customers, and hence a decrease in the demand for both Internet and backbone services. However, the impact on the prices charged by the ISPs only corresponds to the proportion of backbone costs in the total cost of provision of Internet services. If the cost of the backbone represents for example $30 \%$ of the total cost of Internet services, the $10 \%$ increase in the price of backbone services induces an increase in the price of Internet services of only $3 \%$, even if ISPs pass it through entirely.

\footnotetext{
${ }^{20}$ There are at the moment five major backbones: Cable \& Wireless (formerly InternetMCI), MCI WorldCom (including UUnet, ANS, and other previously independent ISPs), Sprint, GTE (including the former BBN and Genuity), and AT\&T (including @Home and the IBM Global Network); Marcus (forthcoming) assesses that "somewhere between six and perhaps thirty other ISPs could also be viewed as Backbone ISPs".

${ }^{21}$ Although this is not represented in our figure, the backbone might have some peering relationships, mostly for historical reasons, with a few ISPs. To conduct the hypothetical monopoly test, we assume that the backbone refuses to accept any increase in traffic from current or potential peers, or alternatively, that it imposes high enough termination charges for increased traffic from current peers or for new traffic from new peers that customers could not avoid the customer price increase by connecting to the peers.
} 
While these comments refer to the reduction in demand by those endusers who are only indirectly connected to the hypothetical monopoly backbone, they similarly apply to end users (web sites, universities, governmental organizations, dedicated access business customers) who may be directly connected to the monopoly backbone and are thus directly confronted with the $10 \%$ price increase. ${ }^{22}$ As in the case of indirect customers, the impact of this price increase on the customer's total cost of Internet services depends on the share of backbone costs in the total cost of Internet services. The methodology is therefore similar for direct and indirect customers.

\subsection{Wholesale-level demand substitution through bi- lateral agreements}

To discuss the various strategies ISPs can use to circumvent the increase in the price of backbone services, let us return to figure 1. Different ISPs are connected to the backbone. We have represented two local markets for end users. In the first one are present ISPs A, B, C and D, while in the other one are present ISPs E, F and G, as well as A and B, which are large national ISPs. Simple arrows represent transit or customer relationships (for the purpose of this paper, we will not distinguish between the two). Thus, in the figure, all the ISPs, except for ISP D are direct customers of our hypothetical backbone monopolist. ISP D connects to the backbone through a customer relationship with ISP C.

Because there is a substantial amount of traffic between ISPs A and B in the western local market, they have entered a local secondary peering relationship, which is represented by the double arrow $\leftrightarrow$. This implies that all local traffic in the western local market between A and B is transmitted directly, presumably through a private interchange.

In this setting, we discuss here circumvention strategies that rely solely on bilateral agreements: transit and local secondary peering. The next subsection discusses more ambitious cooperation among ISPs.

\subsubsection{Purchase of transit}

If the price for backbone services increases as described above, an ISP's first circumvention strategy might be to purchase transit from other ISPs. For instance in figure 2, ISP $\mathrm{F}$ has decided not to connect directly to the backbone, but rather to purchase transit from ISP E. Assuming that the

\footnotetext{
${ }^{22}$ Assuming price increases are not discriminatory. The decrease in demand would be smaller if we made the perhaps more reasonable assumption that the backbone monopolist tailors the level of the price increase to the identity of the customer.
} 


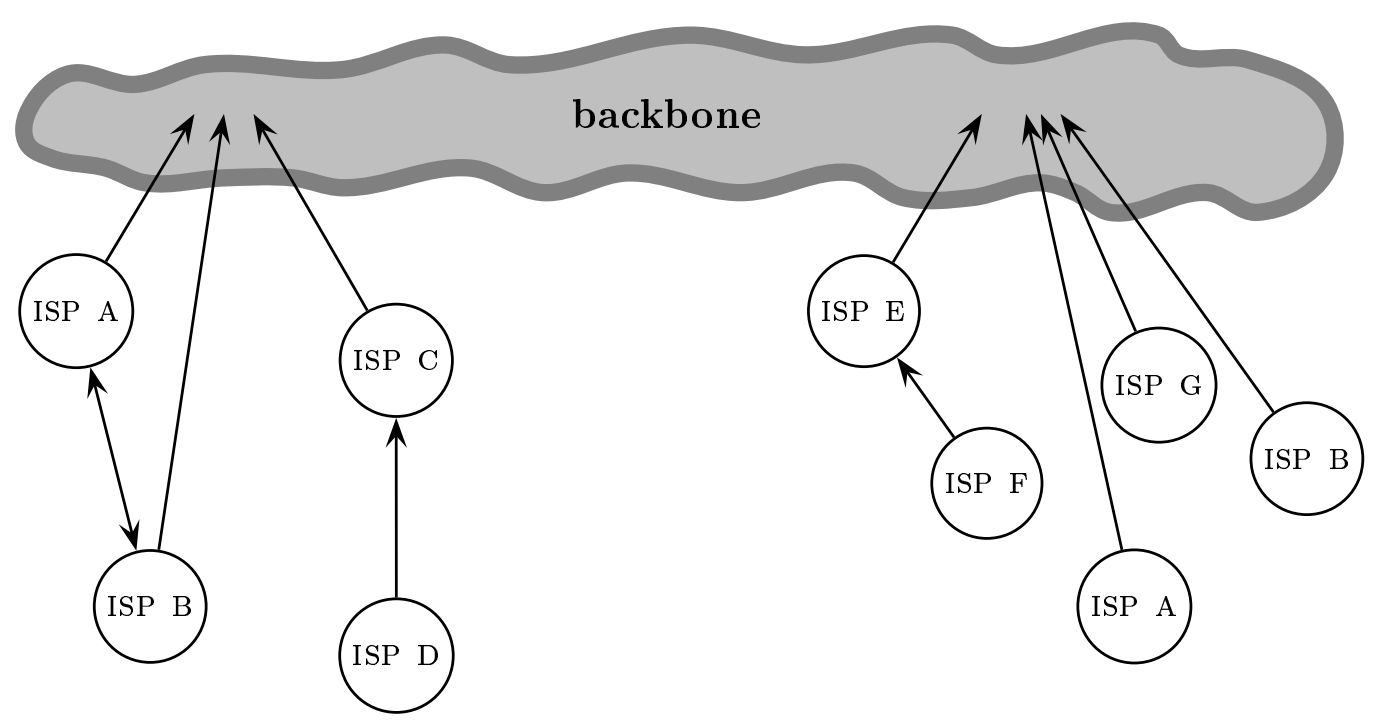

Figure 2: Transit purchase by ISP F.

price increase is passed by $\mathrm{E}$ to $\mathrm{F}$, this will not defeat it: before the price increase, implementing such a transit relationship was not worthwhile, and the comparative cost of direct connection to the backbone is not influenced by a uniform increase in prices. There will be no increase in the purchase of transit due to an increase in the price of backbone services.

\subsubsection{Local secondary peering}

ISPs could also react to the price increase by "secondary peering", that is by building direct peering relationships between themselves. This is represented in figure 3 where ISPs $\mathrm{E}$ and $\mathrm{F}$ enter a secondary peering relationship. Technically, this implies that the (local) traffic between $\mathrm{E}$ and $\mathrm{F}$ is transmitted directly through a private interchange, without going through the backbone. (The peering relationship will also put limits on the traffic that can go through this interchange: it can only be traffic from a client of one of the participating ISPs to a client of the other one.) Large ISPs like A and $\mathrm{B}$, who already had peering relationships on the western market might also react to the price increase by building new peering relationships in other markets, such as the eastern market in the figure.

An increase in the price for backbone services might trigger some secondary peering between ISPs. However, the new secondary peering relationships would be chosen only by ISPs for whom it is currently not rational to 


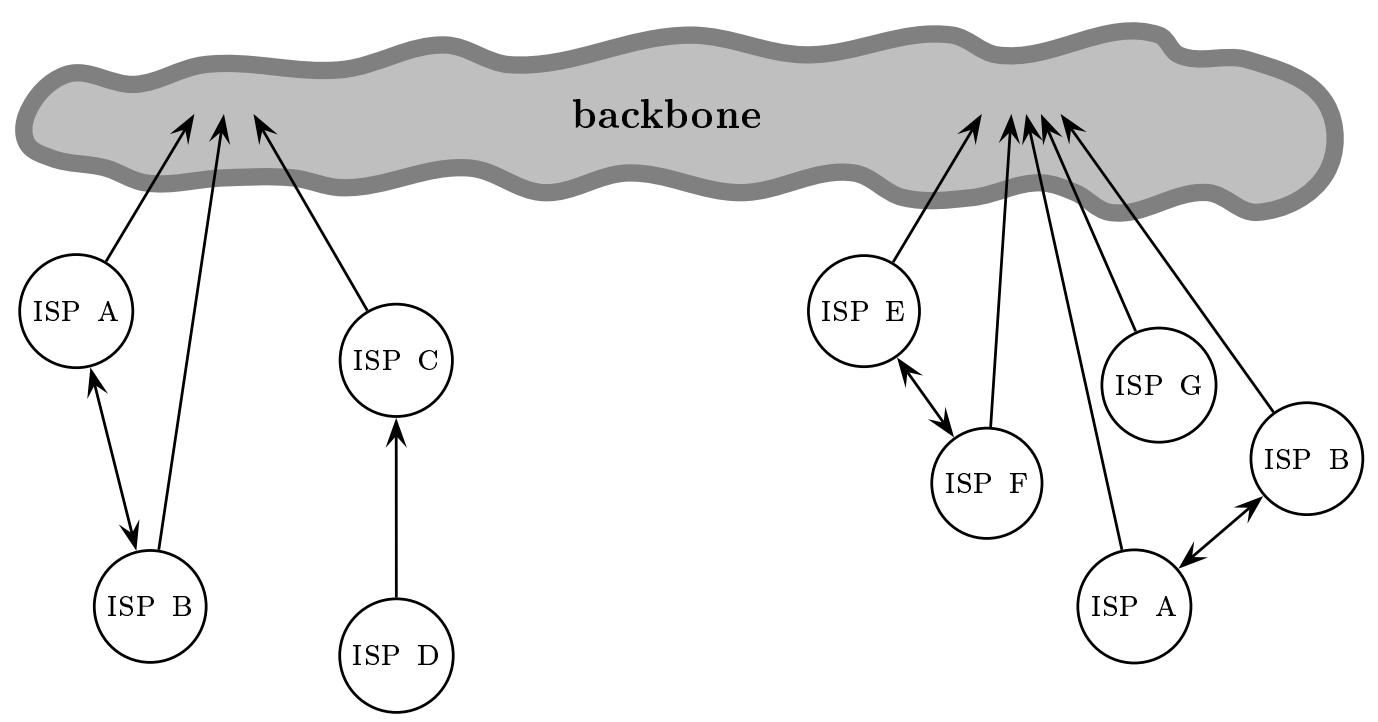

Figure 3: Secondary peering.

peer but for whom the cost of establishing and operating local connections is smaller than the cost of exchanging local traffic through the backbone at the new augmented price. Since local traffic is usually a small fraction of the total traffic, it is likely that local secondary peering would have a limited impact on the demand for backbone services. ${ }^{23}$

\subsection{Cooperation among ISPs for an alternative to the backbone}

A more ambitious circumventing strategy, that might involve substantially more substitution than local secondary peering, would be for some ISPs to create a network among themselves which would offer connectivity for long-haul communications, through a sequence of interconnections. This is represented in figure 4, where the ISPs have created an alternative network through a sequence of peering relationships.

This strategy would meet several obstacles that we now discuss without attempting to quantify them. These obstacles are of two basic types: some of them would make it difficult for the ISPs to organize their network in the short term, the others would make this strategy expensive as a permanent

\footnotetext{
${ }^{23}$ The backbone could furthermore raise the implicit cost of secondary peering by increasing the "volume discount" in its tariffs (that is by affecting the $10 \%$ price increase to the fixed/flat monthly connection fee to the backbone).
} 


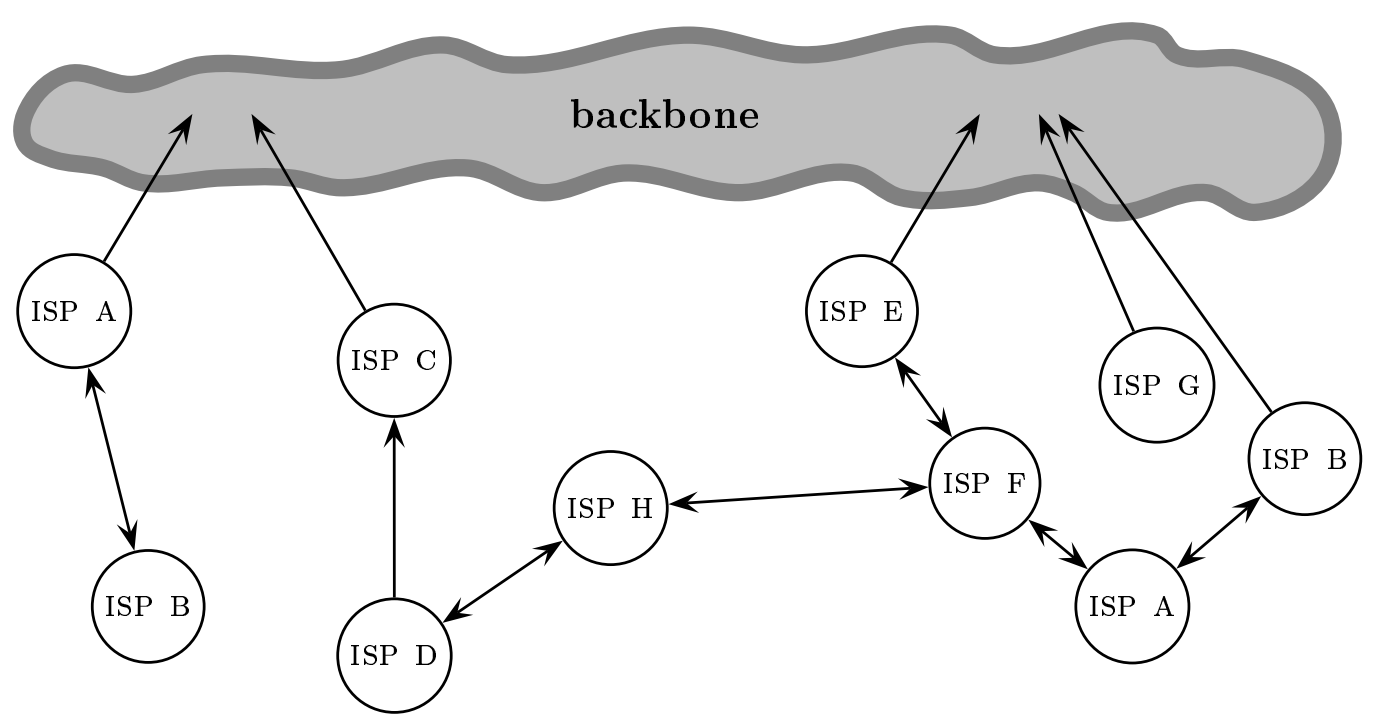

Figure 4: Replacing part of the backbone by a sequence of peering relationships.

business model. We discuss these two aspects in turn.

\subsubsection{Getting the bandwagon rolling}

Coordination A potentially large number of ISPs need to come to an agreement, which raises concerns about coordination. The coordination problem is aggravated by the lead time in building the new network. Setting up the new connectivity structure would involve a lag, since one must build bilateral interconnections and test new routing procedures. This lag might lead the involved ISPs to have concerns about the credibility of the circumvention strategy.

Switching costs Some ISPs have long-term contracts, and switching may require changes in the IP addresses of some of them. The existence of longterm contracts aggravates the coordination problem, while the latter costs reduce the profitability of the circumvention strategy.

Divide and conquer As in Innes \& Sexton (1993), the hypothetical monopolist could make discriminatory offers, that impedes such forms of "backward integration". 


\subsubsection{Organizational issues}

Cost and quality inefficiency Whatever the legal arrangement, the number of interfaces through which packets would transit would increase and delays would also probably increase. Furthermore, the assignment of responsibility for delays would become even more difficult.

The multi-network arrangement may also give rise to gaming, which could make routing inefficient. Last, the transaction costs of dealing with many parties could be large.

Management difficulties in the alternative network Whatever the organizational form, the management of the joint venture would involve a large number of decisions, over which the ISPs might well disagree: financing of expansion of capacity (recall that traffic increases very fast), the location of new capacity, protocols, design and implementation of monitoring capabilities in order to allow the allocation of responsibilities for delayed or lost packets.

It would be difficult to agree on such decisions. Hansmann (1996) and others argue forcefully that heterogeneity hurts associations and joint ventures. In the case of concern here, there are indeed heterogeneous interests due to differences in locations and coverage, in customer mixes, in the size and loyalty of installed bases, and in service offerings.

\subsubsection{Other circumventing strategies}

The ISPs could alternatively form a joint venture and either buy up or enter into an agreement with an existing backbone (one not already included in the group of backbone providers), or build a new backbone. This would greatly ease contracting, as the problem of managing a large number of peering relationships would be simplified. However, the "bandwagon obstacles" mentioned above would remain. Lead time would also be long as the new backbone would need to build a substantial amount of capacity (leasing or adding fiber is not the problem, but hiring (scarce) personnel with the ability to run an IBP and setting up the routers and the interconnection software would be). And some ISPs might not want to become part of the new venture if they doubt that the others will join the parade.

\subsection{Entry into the backbone market}

Relatedly, in response to the price increase, a new backbone, or a little less unlikely, a small backbone not already included in the group, could build up 
capacity. For that new backbone to be credible, it would have to expand its capacity quickly and sign up a substantial fraction of the market. Such an entrant would face important obstacles. ${ }^{24}$

First, it is very costly to build a large network fast (shortage of qualified personnel, lack of learning by doing). Second, the bandwagon obstacles mentioned above would still have to be faced. In particular, given the importance of network externalities, it would be very hard to convince customers to migrate to the new network. Third, the profitability of expansion is problematic. As the new backbone would compete head to head with the hypothetical monopolist, its profits would be small unless it signs up a substantial proportion of customers.

\subsection{Market shares}

Although quantitatively very rough, the analysis above strongly suggests that if the largest backbones merged, then their customers would be better off accepting a 5 to $10 \%$ price increase and keep ubiquitous connectivity rather than trying to cooperate to design a complex and costly circumventing strategy and at the very least lose some connectivity. This holds all the more as the backbone market is currently quite competitive and prices of access to the backbones are low.

When contemplating, say, a merger, competition policy officials must then assess the combined market share of the merging parties. This is no easy task, all the more as market shares can be assessed for example in terms of revenues, traffic, or capacity. For example, in the WorldCom-MCI merger case, the European Commission estimated that backbone market (which they called "top-level ISP") was composed of 16 firms whose total revenues was USD 2,300 million in 1997 and stated that on the basis of revenue: ${ }^{25}$

"WorldCom's share would have been [between 35 and $45 \%$ ] (*), and MCI would have added some [between 5 and 15\%](*), giving the combined group some [between 45 and $55 \%$ ] $(*)$ of the market. Its two nearest competitors would have enjoyed a combined market share of [between 15 and 25\%](*)" 26

\footnotetext{
${ }^{24}$ A strategy for building a new backbone could be to merge and link ISPs. This is the strategy used by Verio, created in March 1996, the Company which has acquired over 45 independent ISPs (see http://www. verio.com). Such a strategy would face the same obstacles as those described in the text.

25 "Parts of this text have been edited to ensure that confidential information is not disclosed; those parts are enclosed in square brackets and marked with an asterisk."

${ }^{26}$ European Commission (1998) at $\oint 104$.
} 
On the basis of traffic flows, the market was very concentrated if the backbone market were considered as being composed of GTE, MCI, Sprint and the WorldCom group, with WorldCom's market share between 50 and $60 \%$ and that of MCI between 15 and $25 \%$, whereas with the other twelve backbones added in, the combined shares of MCI and WorldCom was between 42 and 52\%. (European Commission (1998), at $\S \S 111$ to 113.)

\section{Duopoly}

In this section, we use a highly stylized model of network externalities in order to analyze the competition between two backbones, which differ in the size of their installed bases. We show that the backbone with the larger installed base has a strategic motivation for degrading the quality of interconnection.

\subsection{Demand side}

There are two backbones, $i=1,2$, and a large number of customers, who can be thought of as ISPs, dial-up users or dedicated access customers indifferently, because the only feature of their demand on which we will focus is their preference for connectivity. Backbone $i$ has an installed base of customers $\beta_{i} \geq 0$, with $\beta_{1} \geq \beta_{2}$, and we will refer to backbone 1 as the "bigger" or "dominant" backbone and to backbone 2 as the "smaller" backbone (we will show later on that the bigger backbone also attracts more new customers and thus stays bigger). We assume that the installed bases are locked in previously signed contracts, whose terms cannot be changed by the networks.

There are also new or unattached customers for which the two backbones compete. An unattached customer of type $\tau \in[0,1]$ obtains a net surplus from subscribing to backbone $i$ at price $p_{i}$ equal to

$$
\tau+s_{i} \Leftrightarrow p_{i}
$$

where $s_{i}$, the quality of service of backbone $i$, is given by

$$
s_{i}=v\left[\left(\beta_{i}+q_{i}\right)+\theta\left(\beta_{j}+q_{j}\right)\right],
$$

with $q_{i}$ the number of unattached customers enrolled by backbone $i, q_{j}$ the number of unattached customers signed up by the rival backbone, ${ }^{27} \theta \in[0,1]$ the quality of interconnection (we discuss this in more detail in 5.3), and $v$ a parameter that reflects the importance of connectivity.

\footnotetext{
${ }^{27}$ More generally, when the symbols $i$ and $j$ are both used in an equation, $j$ will be assumed different from $i$.
} 
We assume that the parameter $\tau$ is uniformly distributed, which is equivalent to assuming that the demand curve is linear. We normalize the total population of new customers to 1 and, in order to ensure the existence of a stable equilibrium, we assume

$$
v<\frac{1}{2}
$$

Higher values of $v$ would imply tipping effects and instability.

\subsection{Supply side}

\subsubsection{Capacities and prices}

Following Katz \& Shapiro (1985), backbones compete à la Cournot over unattached consumers: they choose their capacities for market expansion simultaneously. Given these capacities, prices adjust at levels such that a) consumers are indifferent between the two backbones and b) demand is equal to supply. Backbones charge monthly subscriber fees but do not price usage. This assumption, which is made for tractability, is consistent with the modelling of the demand side, where the consumer gross surplus depends only on the number of on-net and off-net communication links not on the intensity of their usage. In practice, the utility of a customer and fees depend on usage; we do not believe that taking this fact into account would qualitatively change our results.

A priori unattached customers view the two backbones as perfect substitutes, thus, if both ${ }^{28}$ of them attract new customers, the "quality-adjusted prices" is the same:

$$
p_{1} \Leftrightarrow s_{1}=p_{2} \Leftrightarrow s_{2}=\hat{p} .
$$

The marginal customer, who is indifferent between using the Internet (with either backbone, from (2)) and not using it, has valuation $\tau=\hat{p}$. From the uniform distribution assumption, we obtain

$$
q_{1}+q_{2}=1 \Leftrightarrow \hat{p} .
$$

Together, equations (1), (2) and (3) determine the equilibrium prices $\left(p_{1}, p_{2}\right)$ as functions of the capacities $\left(q_{1}, q_{2}\right)$ : for $i \neq j=1,2$,

$$
\begin{aligned}
p_{i} & =1 \Leftrightarrow\left(q_{i}+q_{j}\right)+s_{i} \\
& =1+v\left(\beta_{i}+\theta \beta_{j}\right) \Leftrightarrow(1 \Leftrightarrow v) q_{i} \Leftrightarrow(1 \Leftrightarrow \theta v) q_{j} .
\end{aligned}
$$

\footnotetext{
${ }^{28}$ We will provide conditions under which this is indeed the case, but we are also interested in the possibility of "corner solutions" in which one of the backbones does not attract any new customer.
} 
Last, we will assume that backbones incur a cost $c$ from connecting each additional customer.

\subsubsection{Connectivity}

To analyze the backbones' decisions on the quality of interconnection, we now consider the following game:

1. Each backbone $i$ chooses a quality $\theta_{i}$ for its "side" of the connection; the quality $\theta$ of the interconnection is then equal to $\min \left\{\theta_{1}, \theta_{2}\right\} .^{29}$

2. Given the quality determined in stage 1, the backbones choose their capacities and prices are determined as above by (4).

We assume that the cost for backbone $i$ of a connectivity level $\theta_{i}$ is $F\left(\theta_{i}\right)$ where $F$ is a nonnegative and weakly increasing function. Let $\theta_{i}^{*}$ denote the preferred connectivity level of firm $i$. As described in the above timing, we posit that the equilibrium connectivity is $\min \left\{\theta_{1}^{*}, \theta_{2}^{*}\right\} .{ }^{30}$ We show here that, as is usually the case in models with network externalities, the larger firm prefers a lower degree of connectivity than the smaller firm, so that in equilibrium $\theta=\theta_{1}^{*} \leq \theta_{2}^{*}$. Furthermore, since backbone 1's preferred level of connectivity decreases when its size increases, connectivity is lower the more dominant the large backbone.

Alternatively, $\theta$ could be determined through a bargaining process in which a firm would subsidize the other in order to provide a higher level of connectivity. If $\min \left\{\theta_{1}^{*}, \theta_{2}^{*}\right\}$ prevails in the absence of agreement then, and assuming for example that a generalized Nash bargaining solution prevails, the backbones would choose a level of connectivity $\theta$ and a payment $t$ from the small backbone to the bigger one which maximize

$$
\begin{aligned}
& \left\{\left[\left(\pi_{1}(\theta) \Leftrightarrow F(\theta)+t\right] \Leftrightarrow\left[\pi_{1}\left(\min \left\{\theta_{1}^{*}, \theta_{2}^{*}\right\}\right) \Leftrightarrow F\left(\min \left\{\theta_{1}^{*}, \theta_{2}^{*}\right\}\right)\right]\right\}^{\xi}\right. \\
& \quad \times\left\{\left[\left(\pi_{2}(\theta) \Leftrightarrow F(\theta) \Leftrightarrow t\right] \Leftrightarrow\left[\pi_{2}\left(\min \left\{\theta_{1}^{*}, \theta_{2}^{*}\right\}\right) \Leftrightarrow F\left(\min \left\{\theta_{1}^{*}, \theta_{2}^{*}\right\}\right)\right]\right\}^{1-\xi},\right.
\end{aligned}
$$

where $\pi_{i}(\theta)$ denotes the profit of backbone $i$, gross of the connectivity cost, and $\xi$ and $1 \Leftrightarrow \xi$ are the relative bargaining powers of backbones 1 and 2 .

\footnotetext{
${ }^{29}$ This technological assumption translates into a strong complementarity between the investments of the two backbones in improving connectivity.

${ }^{30}$ In a pure-strategy equilibrium, both backbones choose the same $\theta_{i}$, and it is well known that such a game can have many equilibria: if firm $i$ expects firm $j$ to choose $\theta_{j}$, it has no reason to choose a $\theta_{i}$ greater than $\theta_{j}$. Because firm $j$ makes the same reasoning the two firms can coordinate on any $\theta \leq \min \left\{\theta_{1}^{*}, \theta_{2}^{*}\right\}$. From the viewpoint of the two firms, $\theta<\min \left\{\theta_{1}^{*}, \theta_{2}^{*}\right\}$ is inefficient, and we will assume that they coordinate on $\min \left\{\theta_{1}^{*}, \theta_{2}^{*}\right\}$.
} 
The former, "no transfer" assumption seems appropriate whenever it is difficult to write a contract that fixes the level of connectivity. In contrast, if it is easy to write such a contract and to monitor compliance, we would expect parties to come to an agreement about the level of connectivity, validating the latter, "compensating transfer" assumption. For simplicity, we focus here on the no-transfer case, but proposition 3 on page 28 shows that the qualitative conclusions carry over to the compensating-transfer case.

Last, we should note that the assumption that the cost of connectivity is a function solely of the connectivity parameter oversimplifies the reality. We will discuss this point in more detail in section 5.3.

\subsection{Equilibrium}

Given that the profit associated with the installed base is constant, backbone $i$ chooses $q_{i}$ so as to maximize its gross profit

$$
\left(p_{i} \Leftrightarrow c\right) q_{i}=\left[1+v\left(\beta_{i}+\theta \beta_{j}\right) \Leftrightarrow(1 \Leftrightarrow v) q_{i} \Leftrightarrow(1 \Leftrightarrow \theta v) q_{j} \Leftrightarrow c\right] q_{i} .
$$

For a given level of connectivity $\theta$, and the equilibrium quantities are $^{31}$

$$
q_{i}^{*}=\frac{1}{2}\left(\frac{2(1 \Leftrightarrow c)+v(1+\theta) \beta}{2(1 \Leftrightarrow v)+(1 \Leftrightarrow \theta v)}+\frac{(1 \Leftrightarrow \theta) v \Delta_{i}}{2(1 \Leftrightarrow v) \Leftrightarrow(1 \Leftrightarrow \theta v)}\right),
$$

where $\beta=\beta_{1}+\beta_{2}$ is the total installed base and $\Delta_{1}=\Leftrightarrow \Delta_{2}=\beta_{1} \Leftrightarrow \beta_{2} \geq 0$ the difference between the sizes of the two backbones' installed bases (to simplify notation, we will sometimes write $\Delta$ for $\Delta_{1}$ ).

Increasing the quality of the interconnection has two effects: a demand expansion effect and a quality differentiation effect. Other things being equal, a better connectivity benefits consumers and, in equilibrium, total demand also increases: ${ }^{32}$

$$
q_{1}^{*}+q_{2}^{*}=\frac{2(1 \Leftrightarrow c)+v(1+\theta) \beta}{2(1 \Leftrightarrow v)+(1 \Leftrightarrow \theta v)} .
$$

However, although new customers a priori view the two backbones as perfect substitutes, backbone 1, who has a bigger installed base, benefits

\footnotetext{
${ }^{31}$ The equilibrium is stable (in the usual "tâtonnement" meaning) if $v<1 / 2$, as this implies that the slope of the reaction functions is smaller than 1 for any $\theta$ in $[0,1]$.

${ }^{32}$ This implies that equilibrium quality-adjusted price $\hat{p}^{*}=\widehat{\tau}^{*}=1-\left(q_{1}^{*}+q_{2}^{*}\right)$ also decreases, that is, unattached consumers still benefit in equilibrium from a better connectivity.
} 
from a better perceived relative quality ${ }^{33}$ when connectivity is not perfect. As a result, it also becomes dominant on the market for new customers as

$$
q_{1}^{*} \Leftrightarrow q_{2}^{*}=\frac{(1 \Leftrightarrow \theta) v \Delta}{2(1 \Leftrightarrow v) \Leftrightarrow(1 \Leftrightarrow \theta v)}
$$

is strictly positive ${ }^{34}$ except when $\theta=1$.

Up to the constant profit from the installed base and the connectivity cost, the equilibrium profits are given by

$$
\pi_{i}^{*}=(1 \Leftrightarrow v)\left(q_{i}^{*}\right)^{2},
$$

and thus covary with the equilibrium quantities. The bigger backbone benefits from the quality differentiation effect, which it trades off against the demand expansion effect, as can be seen on equation (5). The first term is the same for both backbones: it corresponds to the demand expansion effect and increases with $\theta$. The second term stems from the difference in installed bases: it also increases for the small backbone but decreases for the big one when connectivity is enhanced. The greater the difference in installed bases, the greater backbone 1 gains in comparative quality when $\theta$ decreases and thus, the lower its desired level of connectivity.

Therefore, the bigger backbone has less incentives to maintain connectivity, and its incentives further decrease when the difference in installed bases increases. Since the bigger backbone de facto sets the industry-level of connectivity, we can establish:

Proposition 1 The larger backbone prefers a lower quality of interconnection than the smaller backbone: $\theta_{1}^{*} \leq \theta_{2}^{*}$. Moreover, its preferred quality of interconnection decreases when its advantage in installed base increases: keeping the total installed base $\beta$ constant, $\theta_{1}^{*}$ is a (weakly) decreasing function of $\Delta$.

Proof. Up to the constant profit from the installed base, backbone $i$ 's

\footnotetext{
${ }^{33}$ Models of vertical quality differentiation were first studied by Gabszewicz \& Thisse (1979) and Shaked \& Sutton (1982).

${ }^{34}$ For $\theta$ small and $v$ large enough (namely, when $v>1 /(3-2 \theta)$ ), the difference in number of new susbscribers actually exceeds the difference in installed bases $\left(q_{i}^{*}-q_{j}^{*}>\right.$ $\beta_{i}-\beta_{j}$ ).
} 
profit is $\pi_{i}^{*} \Leftrightarrow F$, and simple computations show that ${ }^{35}$

$$
\frac{\partial^{2}}{\partial \theta \partial \Delta_{i}}\left[\pi_{i}^{*} \Leftrightarrow F\right]=(1 \Leftrightarrow v) \frac{\partial^{2}\left(q_{i}^{*}\right)^{2}}{\partial \theta \partial \Delta_{i}}<0 .
$$

The conclusions then follow from a standard revealed preferences argument (using the fact that $\Delta_{1}>0>\Delta_{2}$ ).

The trade-off between the demand expansion effect and the quality differentiation effect is best seen when connectivity is costless $(F(\theta)=0)$. In that case, the smaller backbone always prefers a perfect connectivity $\left(\theta_{2}^{*}=1\right)$ while the bigger backbone either prefers perfect connectivity (if its installed base superiority is small) or no connection at all $\left(\theta_{1}^{*}=0\right)$; the bigger backbone is more likely to choose not to interconnect when the network externality is large ( $v$ close to its maximum value of $1 / 2$ ) and less likely to do so if the total size of the installed base is large ( $\beta$ large, so that the demand expansion effect of a better connectivity is important):

Proposition 2 If $F(\theta)=0$ for all $\theta$ :

i. the equilibrium quality of interconnection is either 0 or 1 ;

ii. for a given total installed base $\beta$, there exists a threshold $\Delta^{*}$ such that the equilibrium quality of interconnection is 1 if $\Delta<\Delta^{*}$ and 0 if $\Delta>\Delta^{*}$. The threshold $\Delta^{*}$ a) increases when the total installed base $\beta$ increases and b) decreases when either the marginal cost $c$ or the magnitude of network externalities $v$ increases.

Proof. See Appendix.

If the quality of interconnection is costly, perfect connectivity $(\theta=1)$ is in general neither socially efficient nor privately optimal, even for the smaller backbone. However, from Proposition 1, the larger backbone still prefers a lower interconnection quality than the smaller backbone. Moreover, the dominant backbone's best strategy is still to refuse interconnection if it is optimal to do so in the absence of a cost of quality of interconnection.

In any event, the level of connectivity is lower than what would be socially desirable, since the bigger backbone (which determines the industry level of

\footnotetext{
${ }^{35} q_{i}^{*}$ is of the form $\psi(\theta)+\rho(\theta) \Delta_{i}$, where both $\rho$ and $\psi \rho$ are decreasing functions of $\theta$. Therefore

$$
\frac{1}{2} \frac{\partial^{2}\left(q_{i}^{2}\right)}{\partial \theta \partial \Delta_{i}}=\left(\psi^{\prime}+\rho^{\prime} \Delta_{i}\right) \rho+\left(\psi+\rho \Delta_{i}\right) \rho^{\prime}=(\psi \rho)^{\prime}+2 \rho \rho^{\prime} \Delta_{i}<0 .
$$
}


connectivity) takes into account the impact of its interconnection decision neither on its rival, whose profit increases with the level of connectivity, nor on consumers, who also benefit from an improved connectivity in the relevant case where $\theta_{1}^{*}>0$ :

- unattached consumers always benefit from a better connectivity, since the quality-adjusted price $\hat{p}$ decreases with $\theta$;

- installed-base consumers also benefit from a better connectivity, since it provides a better access to the other backbone's consumers and moreover attracts additional consumers on both backbones. ${ }^{36}$

An increase in the bigger backbone's installed base advantage reduces its incentives to maintain connectivity, thus exacerbating the divergence between social welfare and private incentives and leading to a quality of interconnection further below the socially desirable level.

\section{$5 \quad$ Modeling assumptions}

We now discuss the robustness of our analysis to the modeling assumptions.

\subsection{Negotiated connectivity}

We have assumed that the equilibrium connectivity is equal to the level set by the network that values connectivity the least. Let us briefly analyze the opposite polar case in which the networks can contract on the quality of their interconnection before waging product market competition. As discussed above, under these circumstances, the smaller network in general bribes the larger network to accept a higher level of connectivity. (Alternatively, and probably more realistically, the smaller network can undertake a disproportionate fraction of the interconnection investment. ${ }^{37}$ ) From a social welfare

${ }^{36}$ Backbone $i$ 's installed-base consumers enjoy network externalities

$$
v\left[\left(\beta_{i}+q_{i}^{*}(\theta)\right)+\theta\left(\beta_{j}+q_{j}^{*}(\theta)\right)\right]
$$

and an increase in $\theta$ always increases $q_{2}^{*}$. If $\theta_{1}^{*}>0$ then, since $\theta_{1}^{*}$ maximizes $(1-v)\left(q_{1}^{*}(\theta)\right)^{2} / 4-F(\theta)$ it must be the case that $q_{1}^{*}\left(\theta_{1}^{*}\right) \geq q_{1}^{*}(0)$ (since $F$ is nondecreasing), and thus that any level $\theta$ higher than $\theta_{1}^{*}$ attracts more new customers than any lower level (since $q_{1}^{*}$ is convex in $\theta$ ).

${ }^{37}$ In reality, it could very well be that the size of the bribe that has to be paid is greater than the total cost of connectivity. The analysis that follows assumes that in this case the backbones find some other way to transfer funds between each other. 
perspective, though, the relevant variable is the resulting level of connectivity. This level, $\theta^{*}$, maximizes joint profit: ${ }^{38}$

$$
\max _{\theta}\left\{\pi_{1}(\theta)+\pi_{2}(\theta) \Leftrightarrow 2 F(\theta)\right\}
$$

Intuitively, the negotiated level of connectivity will be intermediate between what would be optimal for each backbone. Less obvious a priori is the impact of the difference in installed bases, since a larger difference increases the smaller backbone's demand for connectivity but further reduces the bigger backbone's one. However, an increase in $\Delta$ decreases the preferred connectivity of the bigger backbone more than it increases the preferred connectivity of the smaller backbone, and therefore a larger asymmetry between the two installed bases reduces the negotiated level of connectivity. The reason is that a low level of connectivity increases the "vertical differentiation" between the two networks and thereby softens product market competition.

Proposition 3 The negotiated level of connectivity, $\theta^{*}$, satisfies $\theta_{1}^{*} \leq \theta^{*} \leq$ $\theta_{2}^{*}$ and $d \theta^{*} / d \Delta \leq 0$.

Proof. See Appendix.

The qualitative conclusions obtained when assuming that connectivity is noncontractable are therefore still valid when connectivity is contractable and freely negotiated by the parties: the negotiated level of connectivity, which lies somewhere between the two parties' privately optimal levels, decreases when the bigger backbone's installed base advantage increases.

\subsection{Modeling of competition}

The Cournot-cum-installed-bases model employed in this paper is highly stylized. Its tractability would be no justification for using it if we did not believe that, despite its limitations, it yields the "right picture". We should therefore discuss the robustness of our conclusions to the modeling of competition. The assumptions of the foregoing model may understate in some ways and overstate in others the difficulty faced by a smaller player in resisting to a dominant firm.

\footnotetext{
${ }^{38}$ Any efficient bargaining process will lead the parties to maximize their joint profit and to divide it according to their bargaining power. For example, the outcome of the generalized Nash-solution suggested in the previous section consists of the joint-profit maximizing level of connectivity and a transfer from the smaller backbone to the bigger one; this transfer increases with the bargaining weight $\xi$ of the bigger backbone.
} 
A couple of assumptions stack the deck against the possibility of the extension of market dominance. First, we have imposed an upper bound on the magnitude of network externalities by requiring equilibria to be "stable". Larger network externalities would give rise to "tipping effects" and make it more likely that the industry would be monopolized.

Second, and relatedly, we have assumed that networks compete in their capacities to enlist and serve new subscribers. Were capacities to adjust fast as a response to increases in demand, Bertrand competition would be a more appropriate assumption than Cournot competition. Bertrand competition gives rise to stronger tipping effects. We have tested the robustness of our conclusions by exploring an alternative model where the two (now horizontally differentiated) backbones compete in prices rather than in capacities, and shown that the same conclusions apply. The larger backbone has still less incentives to invest in the quality of interconnection; and, assuming away the costs of interconnection, a small backbone always favors the highest possible interconnection quality, whereas the dominant backbone may prefer a lower quality level, and even $\theta=0$.

In contrast, we have assumed that installed bases are locked in. Section 2.4 discussed at length the various psychological, physical and contractual switching costs in this industry. Yet, the assumption that the installed bases are locked in is too strong. While installed base customers would not switch in our equilibrium (even for negligible switching costs), with moderate switching costs, a fraction of them might be poached by very aggressive offers by the backbones. We do not feel, however, that this would affect our conclusions in any substantial way. Moderate switching costs constitute in fact a mixed blessing for a small player, as they make its installed base an easy target for a large backbone that degrades interconnection. A finer modeling of installed base behavior would be interesting in its own right, but in the context of our analysis, it would complicate substantially the description of equilibrium without, we believe, altering the main insights.

\subsection{Modeling the quality of interconnection}

In this subsection, which can be omitted without loss of continuity, we discuss two interpretations of the parameter $\theta$ : compatibility of standards and interface capacity.

The first interpretation corresponds to a compatibility decision. Compatibility levels however may be intermediate between 0 and 1, as connection standards may allow some services but not others. For instance, the backbones may be compatible for standard Internet usage, but not for some or all the new, delay-sensitive services such as Internet telephony or video- 
conferencing, because one of the backbones refuses to offer premium interconnection services to the other or develops proprietary standards.

The second interpretation of the quality parameter $\theta$ refers to the capacity of the interface (or of the links near the interface). A lower capacity translates into delays and losses of packets.

To illustrate this, consider the following model of "discouragement", where the gross utility of a connection, $w$, is randomly distributed according to a distribution $F($.$) ; the parameter v$ can in that case be reinterpreted as the expected gross utility:

$$
v=E[w]=\int_{0}^{+\infty} w d F(w) .
$$

The utility is equal to the expected gross utility minus the average delay at the interface. Assuming that the interface has capacity $\mu$ and faces a traffic $t$, and positing a M-M-1 process for interface traffic, the average disutility of delay is $k /(\mu \Leftrightarrow t)$, where $k$ is a constant. Given the existence of a delay, only those connections with gross utility $w \geq w^{*}$ will actually be made, with

$$
w^{*}=\frac{k}{\mu \Leftrightarrow t} .
$$

The off-net traffic is therefore

$$
t=2\left(\beta_{1}+q_{1}\right)\left(\beta_{2}+q_{2}\right)\left[1 \Leftrightarrow F\left(w^{*}\right)\right] .
$$

Conditions (7) and (8) together define the threshold $w^{*}$ as a function of the capacity $\mu$ and of the potential exchange traffic, $T=2\left(\beta_{1}+q_{1}\right)\left(\beta_{2}+q_{2}\right)$. Using equation (1), the average value for a customer of backbone $i$ with type $\tau$ is

$$
\tau+v\left(\beta_{i}+q_{i}\right)+\theta\left(\beta_{j}+q_{j}\right),
$$

where the "quality" of the interconnection,

$$
\theta=\frac{\int_{w^{*}}^{+\infty}\left(w \Leftrightarrow w^{*}\right) d F(w)}{\int_{0}^{+\infty} w d F(w)}
$$

is now endogenous and depends positively on the capacity $\mu$ but negatively on traffic and thus on the two backbones' customer bases. If the interface capacity is infinite $(\mu=+\infty)$, then $w^{*}=0$ and (9) coincides with (1) with $\theta=1$ (perfect interconnection), whereas if the interface capacity is equal to zero $(\mu=0)$, then $w^{*}=+\infty$ and (9) coincides with (1) with $\theta=0$. 
More generally, given $\left(q_{1}, q_{2}\right)$, the choice of interface capacity $\mu \in[0,+\infty)$ determines a quality of interconnection $\theta \in[0,1]$, with a higher $\mu$ generating a higher $\theta .^{39}$

This modified model with volume-dependent interconnection quality has the same flavor as the basic one, but is mathematically more complex. Each backbone's marginal profit with respect to output includes an extra, nonlinear term, which represents the marginal impact of adding one more consumer to one's network on the quality of interconnection. In a static setting such as the one we consider here, the thrust of the analysis is the same as before and amounts to focusing on the strategic choice of the interface capacity $\mu$. The additional effect, which could be labelled the "marginal degradation cost of output expansion", however induces backbones to reduce their expansion relative to a situation in which their output has no impact on interface congestion.

The marginal degradation costs is not the same for both backbones. Indeed, an increase in the size of the bigger backbone degrades the interface quality less than an increase in the size of the smaller backbone, since a higher fraction of messages issued by customers of the smaller backbone are directed off net. ${ }^{40}$ Another interesting implication of the marginal degradation term is that even equal-size backbones may want to restrict the capacity of their interface in order to reduce the intensity of competition for new customers. Last, tipping need not arise even if network externalities are strong $(v>1 / 2)$. When market shares become quite unequal, off-net traffic is reduced and so the quality of interconnection is restored (provided the interface capacity is not too small), which counteracts the tendency toward tipping.

\footnotetext{
${ }^{39}$ The endogeneity of $\theta$ thus provides an additional way for the dominant backbone to alter the quality of the interface: it can degrade this quality not only by reducing the interface capacity $\mu$, but also by increasing its customer base $\beta_{1}+q_{1}$.

${ }^{40}$ Using (9), this term, per new customer of network $i$, is

$$
d_{i}=\left(\beta_{j}+q_{j}\right)\left[1-F\left(w^{*}\right)\right] \frac{\partial w^{*}}{\partial q_{i}}<0 .
$$
}

Using (7) and (8),

$$
\frac{\partial w^{*}}{\partial q_{i}}\left(\beta_{i}+q_{i}\right)=\frac{\partial w^{*}}{\partial q_{j}}\left(\beta_{j}+q_{j}\right)
$$

and so

$$
d_{i}=\frac{\left(\beta_{j}+q_{j}\right)^{2}}{\left(\beta_{i}+q_{i}\right)^{2}} d_{j} .
$$




\section{Oligopoly and targeted degradation}

The preceding analysis emphasized the key consequences of the difference in the size of the installed bases: the bigger backbone has an incentive to degrade the quality of interconnection. Building on this insight, we now analyze the possible adverse effect of a merger that creates a "large" backbone, starting from an initial situation where backbones are symmetric in size. Specifically, we assume that, initially, four equal-sized backbones ${ }^{41}\left(\beta_{i}=\beta / 4\right)$ compete for new customers. We briefly analyze the competition between these four backbones, and then study the outcome of a merger between two of them: a merger between backbones 1 and 4 creates a "dominant backbone", which we will label backbone 1 , with an installed base of size $\beta / 2$, competing with two smaller backbones (backbones 2 and 3), each with an installed base of size $\beta / 4$.

For computational simplicity, we assume that each backbone may unilaterally and costlessly choose to be compatible with each of the other backbones $\left(\theta_{i j}=0\right.$ or 1 , and $\left.F(1)=F(0)=0\right)$. As above, compatibility requires cooperation of both parties: the effective level of connectivity between two backbones is the minimum of the levels chosen by each backbone. Last, and as we will later discuss in some detail, we will allow the backbones to limit the traffic at the interfaces even when they are compatible.

We now successively consider the no-merger situation and the alternative post-merger strategies.

\subsection{No merger: Competition among equals}

We first show that backbones have an incentive to maintain the quality of interconnection in the pre-merger situation. Since each backbone has a smaller installed base than the combined installed base of its rivals, maintaining a high quality of interconnection has a positive impact on both the demand (demand expansion effect) and on its relative perceived quality, compared with that of the rivals (quality differentiation effect).

With four equal-size backbones competing with each other, the analysis

\footnotetext{
${ }^{41}$ We have chosen this initial situation in part because it bears a vague ressemblance to the actual backbone market with its four dominant players, but also for analytical convenience. Furthermore, with four backbones the effect of a merger is to create a large backbone with a still moderate market share, so that our results are not biased towards degradation of connectivity. A more "realistic" analysis would not only take into account the difference in the sizes of the networks, but also the presence of a competitive fringe.
} 
is similar to that of the duopoly case and the profit of backbone $i$ is

$$
\pi_{i}=\left[1 \Leftrightarrow q_{i} \Leftrightarrow \sum_{j \neq i} q_{j}+v\left(\frac{\beta}{4}+q_{i}+\sum_{j \neq i} \theta_{i j}\left(\frac{\beta}{4}+q_{j}\right)\right) \Leftrightarrow c\right] q_{i} .
$$

As before, decreasing the quality of interconnection with backbone $j$ has two effects on backbone $i$. It decreases total demand, because both backbones provide access to fewer customers. It also lowers the quality of backbone $i$ compared to the quality of the other two backbones, while not affecting the quality difference with backbone $j$. As a consequence, in the absence of cost saving, there are no incentives to degrade the interconnection with a single other backbone.

Similarly, degrading interconnection with several backbones is not profitable, as the demand reduction is even larger than before, and the quality differentiation effect still plays against the degrading backbone which offers access to a smaller fraction of the installed base than the others. As a result, no backbone has an incentive to degrade connectivity:

Proposition 4 In the absence of a merger, all backbones prefer a high quality of connection: $\theta_{i j}^{*}=1$ for all $i$ and $j$. They obtain identical profits $\pi_{i}^{*}=(1 \Leftrightarrow v)\left(q_{i}^{*}\right)^{2}$, with

$$
q_{i}^{*}=\frac{1 \Leftrightarrow c+v \beta}{5(1 \Leftrightarrow v)} .
$$

Proof. See appendix C.

\subsection{Possible strategies for a dominant backbone}

In the post-merger situation the two small backbones still have incentives to maintain a good connectivity but the dominant backbone may not want to. To analyze this issue, we consider three possible strategies for the dominant backbone: ${ }^{42}$

Accommodation:The dominant backbone maintains a good interconnection with the other two backbones;

Global degradation: The dominant backbone decides not to interconnect at all;

\footnotetext{
${ }^{42}$ The analysis and the conditions derived below are consistent with the requirement that equilibria are stable.
} 
Targeted degradation: The dominant backbone refuses interconnection with one of the small backbones and furthermore limits the interface capacity with the other backbone, to discourage it from offering transit services - that is, from transmitting messages from the targeted backbone's clients to the dominant backbone.

Our analysis shows that the merger creates incentives to pursue a targeted degradation strategy. A global degradation strategy is not profitable: it reduces again the demand and does not yield any competitive advantage to the new entity over its rivals, whose combined installed base has the same size as the new entity's. But a targeted degradation strategy can be profitable. If the other backbone does not provide any transit services, the degradation strategy creates an asymmetry between the dominant backbone (who can offer access to three-fourth of the installed base) and the target (who can offer access to only half of the installed base); this particular degradation strategy thus generates a positive quality differentiation effect for the dominant backbone, which can be sufficient to make the strategy profitable. Moreover, by limiting its interface capacity with the non-targeted backbone, the dominant backbone can effectively discourage this backbone from providing transit services to the target.

Constraining the interface with the non-targeted backbone: an illustration. To illustrate the limitation of the interface with the non-targeted backbone, let us return to the interpretation of the interconnection quality parameter as the interface capacity (see section 5.3). Assume for simplicity that all messages have the same value $v$ (so $w \equiv v$ ). Let $\mu$ denote the capacity of the interface and $t_{i j}$ denote the potential traffic between backbones $i$ and $j\left(t_{i j} \equiv 2\left(\beta_{j}+q_{j}\right)\left(\beta_{j}+q_{j}\right)\right)$. Suppose that $t_{12}+t_{13} \geq \mu>t_{12}$, and that network 1 refuses to interconnect with network 3 . In the absence of transit, the value of a message net of the delay cost is $v \Leftrightarrow k /\left(\mu \Leftrightarrow t_{12}\right)$, which is close to $v$ (that is,$\theta=1 \Leftrightarrow k /\left[v\left(\mu \Leftrightarrow t_{12}\right)\right]$ is close to 1$)$ if $k$ is small. In contrast, in the presence of transit, the equilibrium total traffic at the interface, $t$, is lower than $t_{12}+t_{13}$ and is such that interconnection with network 1 is worthless: $v \Leftrightarrow k /(\mu \Leftrightarrow t)=0$. In other words, the gain from connectivity is completely dissipated by the customers' competition for sparse interface capacity. Hence, transit is equivalent (in utilities, although not in outcomes) to the absence of interconnection between networks 1 and $2\left(\theta_{12}=0\right)$. 


\subsection{Post-merger: accommodation versus targeted de- gradation}

Building on the previous insights, it is intuitive that the small backbones have no incentives to degrade their connectivity. Similarly, since the large backbone's installed base has exactly the same size as the total installed base of the other two backbones, it is not a profitable strategy to degrade the quality of interconnection with both other backbones:

Proposition 5 After the merger:

i. the small backbones prefer to maintain connectivity;

ii. the dominant backbone does not want to pursue a global degradation strategy.

Proof. See appendix D.

It follows from this proposition that the dominant backbone's relevant strategies consist in either maintaining connectivity with both smaller backbones (accommodation strategy), or selectively degrading connectivity with one of them (targeted degradation strategy). The accommodation strategy increases the total demand (demand expansion effect) but eliminates any competitive advantage that the dominant backbone could derive from its bigger installed base. ${ }^{43}$ In contrast, a targeted degradation strategy potentially gives the dominant backbone a competitive advantage over the targeted backbone, whose installed base is only half the size of the dominant backbone's installed base.

To fix ideas, assume that backbone 1 does not interconnect with backbone $3\left(\theta_{13}=0\right)$ but maintains a high quality of interface with backbone 2 $\left(\theta_{12}=1\right)$ as long as this backbone does not offer transit to backbone 3 - that is, the capacity of this interface is such that its quality is excellent if backbone 2 does not offer transit to backbone 3, but abysmal otherwise. Offering transit would thus amount for backbone 2 to degrade its connection with the dominant backbone and result in the same situation as with the global degradation strategy, where each backbone has access to half of the installed

\footnotetext{
${ }^{43}$ In the Cournot-like set-up of the present model, the dominant backbone gets less than in the pre-merger situation if he adopts the accommodation strategy. This standard feature of Cournot oligopoly models (see Salant, Switzer \& Reynolds (1983)), where quantities are strategic substitute and a merger always benefits rivals but not necessarily the merging firms, may not hold in alternative models. For example, with Bertrand competition among differentiated backbones, prices tend to be strategic complements and a merger can benefit both the rivals and the merging firms.
} 
base customers; it would therefore decrease the demand for the services of backbone 2 and remove any competitive advantage of this backbone vis-à-vis the other ones. In contrast, by refusing transit and thus maintaining a good connectivity with the dominant backbone:

- backbone 2 has access to the entire installed base, since it has a good connection with the other two backbones;

- the dominant backbone has access to three fourths of the installed base, its own customers plus those of backbone 2 ;

- the target, backbone 3, has access to only half of the installed base customers.

This strongly suggests, and the analysis confirms, that in equilibrium backbone 2 prefers not to offer transit services to backbone $3 .^{44}$ Therefore, for the dominant backbone, the targeted degradation strategy has a negative impact on demand but generates a competitive advantage over the targeted backbone. We show in appendix $\mathrm{E}$ that this competitive advantage may be so large that in equilibrium the targeted backbone does not attract any new customer $\left(q_{3}^{*}=0\right)$ and the dominant backbone gains from adopting this targeted degradation strategy.

Proposition 6 If $v \beta /(1 \Leftrightarrow c)$ belongs to the interval $(1,4(1 \Leftrightarrow 2 v) /(1+v))$ (which is not empty if network externalities are large enough, i.e., if $v>1 / 3$ ), then the targeted degradation strategy prevents the targeted backbone from attracting any new customer and is profitable for the dominant backbone.

\section{Proof. See appendix E.}

The targeted degradation strategy can still be optimal for the dominant network even if does not prevent the target from attracting some new consumers. It would be even more appealing to the dominant backbone if the quality of interconnection were costly.

\footnotetext{
${ }^{44}$ This also suggests that backbone 2 may refuse transit even if the dominant backbone does not limit the capacity of its interface: offering transit to backbone 3 would still remove any quality disadvantage for that backbone and thus lead to more effective competition among the three backbones - although backbone 1 would still suffer from a lower perceived quality. A detailed analysis of the transit decision would however require a careful modeling of the negotiation between the two small backbones and is beyond the scope of the present analysis.
} 


\subsubsection{Discussion: the non-targeted backbone's refusal to provide transit}

The reader may object that our analysis is static and might therefore overestimate backbone 2's willingness to provide transit services to the target, as backbone 2 may fear to be the next target for a degradation of connectivity. The dominant backbone could avoid this problem if it could commit itself to maintaining connectivity with the remaining backbone. ${ }^{45}$ Even if backbone 1 cannot commit not to degrade its connection with backbone 2 tomorrow (i.e., cannot sign a non-aggression pact), it may well refrain from targeting backbone 2 once backbone 3 is weakened; indeed, the targeted degradation strategy gives a competitive advantage to backbone 2 and thus dissipates today part of the initial asymmetry in installed bases.

To see this, suppose for example that there are two periods and that all agents (firms and consumers) behave myopically; also, for the sake of the argument, assume that the first-period targeted degradation eliminates backbone 3, who is no longer present in the second period (it may for example be forced to bankruptcy if it fails to attract enough new customers). ${ }^{46}$ Backbone 2 offers a better quality in the first period, since it provides access to all subscribers, whereas backbone 1 does not offer access to backbone 3's installed base customers; its market share among date 1 new customers is therefore higher than that of backbone 1: denoting by $\hat{q}_{i}^{t}$ the equilibrium number of customers of backbone $i$ in period 1 under targeted degradation, we have (see appendix F)

$$
\hat{q}_{2}^{t} \Leftrightarrow \hat{q}_{1}^{t}=\frac{v \beta}{4(1 \Leftrightarrow v)}>0 .
$$

While this does not suffice to erase entirely the difference in installed base, ${ }^{47}$ it does dissipate part of it: both installed bases are larger in the second

\footnotetext{
${ }^{45}$ In that case, it could even auction off the privilege of not being the target in the first period.

${ }^{46}$ Two alternative hypotheses could be made about the fate of the $\beta / 4$ customers in its installe base that backbone 3 liberates:

a. We could assume that backbones 1 and 2 each buy half of them before competing at date 2 . This would reinforce our conclusions as the installed base $\hat{\beta}$ increases.

b. We could assume that these consumers go back on the market at date 2. Then, the analysis is made more complicated by the fact that it would be unreasonable to assume that their types are uniformly distributed on $[0,1]$, since the fact that they have already chosen to connect is proof that they value connectivity.

${ }^{47}$ The no-tipping condition $v<1 / 2$ implies $q_{2}^{t}-q_{1}^{t}<\beta_{1}-\beta_{2}=\beta / 4$.
} 
period $\left(\hat{\beta}_{i}=\beta_{i}+\hat{q}_{i}^{t}\right)$, but their difference is smaller:

$$
\hat{\Delta}=\hat{\beta}_{1} \Leftrightarrow \hat{\beta}_{2}=\frac{1 \Leftrightarrow 2 v}{1 \Leftrightarrow v} \frac{\beta}{4}<\Delta=\frac{\beta}{4} .
$$

Appendix $\mathrm{F}$ shows that the bigger backbone does not have an incentive to degrade connectivity in the second period; that is, $\hat{\Delta}<\Delta^{*}(\hat{\beta})$ where $\Delta^{*}(\hat{\beta})$ is the threshold, characterized in our duopoly analysis, below which degrading connectivity becomes unprofitable for the bigger backbone.

This simple exercise is only meant to convey the intuition. A more rigorous analysis should allow for the fact that firms and consumers take into account the evolution of the industry in their decisions. However, a number of factors suggest that the argument is robust. First, two factors make the degradation of the connection less appealing in the second period: the total installed base is bigger, and the difference in installed base is lower. It can be checked that the latter effect is in fact sufficient to deter the degradation strategy. ${ }^{48}$ Second, backbone 2 can decide to further expand its first-period capacity so as to ensure that degrading connectivity is not optimal for backbone 1 in later periods. To be sure, backbone 1 might choose to counteract this by expanding its own capacity in the first period. However, there is an asymmetry here, since any expansion in capacity in the first period increases the total installed base in the second period, which in turn tends to make a degradation strategy less appealing. ${ }^{49}$ And, more important, backbone 2's incentive to avoid degradation is likely to be higher than backbone 1's incentive to degrade, since degrading connectivity reduces total demand and, therefore, the overall industry profitability. ${ }^{50}$

\footnotetext{
${ }^{48}$ The threshold $\Delta^{*}(\beta)$ increases with $\beta$, as noted in our analysis of the duopoly situation, but we actually have here $\hat{\Delta}<\Delta^{*}(3 \beta / 4)\left(<\Delta^{*}(\hat{\beta})\right)$.

${ }^{49}$ Suppose for example that, starting from a situation where the second-period installed bases $\hat{\beta}_{1}=\beta_{1}+\hat{q}_{1}^{t}$ and $\hat{\beta}_{2}=\beta_{2}+\hat{q}_{2}^{t}$ would exactly satisfy $\hat{\beta}_{1}-\hat{\beta}_{2}=\Delta^{*}\left(\hat{\beta}_{1}+\hat{\beta}_{2}\right)$, backbone 2 increases its capacity $\hat{q}_{2}$ by $\delta$ so as to avoid degradation in the second period; then, backbone 1 would have to increase its own first-period capacity by more than $\delta$ to restore its incentives to degrade connectivity in the second period, since

$$
\left(\hat{\beta}_{1}+\delta\right)-\left(\hat{\beta}_{2}+\delta\right)=\hat{\beta}_{1}-\hat{\beta}_{2}<\Delta^{*}\left(\hat{\beta}_{1}+\hat{\beta}_{2}+2 \delta\right) .
$$

${ }^{50}$ Suppose for example that, due for instance to a larger initial difference in installed bases, $\hat{\Delta}$ is slightly larger than $\Delta^{*}(\hat{\beta})$ if firms behave myopically. Backbone 2 can then expand its capacity in order to move date 2 difference in installed bases in the region $\hat{\Delta}<\Delta^{*}(\hat{\beta})$, where connectivity is maintained in the second period; conversely, backbone 1 can expand its capacity so as to remain in the region where connecticity would be is degraded. However, since aggregate profit jumps up when connectivity is restored,
} 


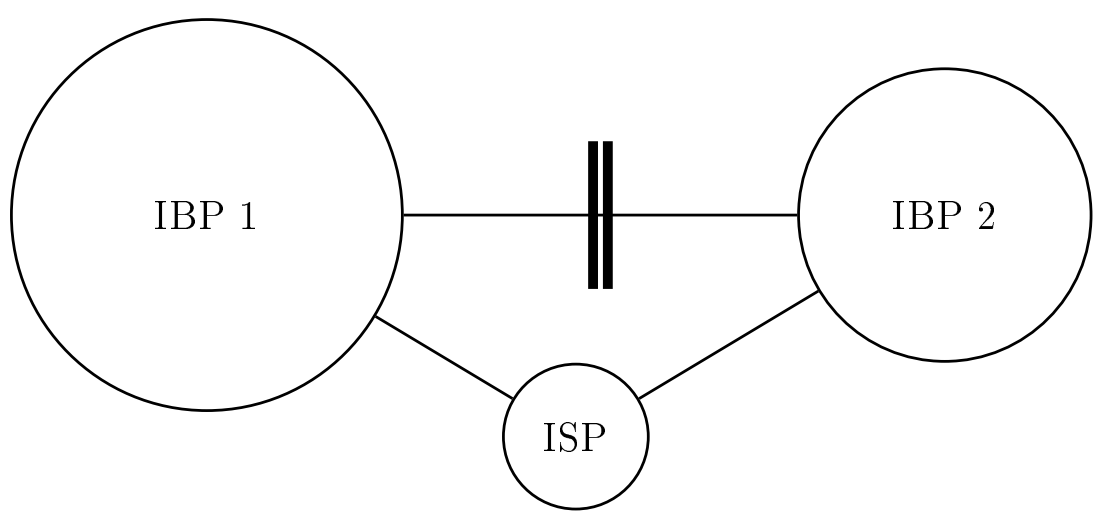

Figure 5: Multihoming

Other reasons may induce backbone 2 to refuse to provide transit services to backbone 3. First, giving transit may impose too much strain on its network. Second, even if it anticipates to be the next target once backbone 3 is no longer an effective competitor, backbone 2 may still prefer to refuse transit and thus weaken backbone 3 , since this yields a much higher profit in the first-period: If it refuses transit, backbone 2 offers the maximal quality of service and is the only one to do so, whereas providing transit services leads to a less profitable situation, where three equally low-quality backbones compete head-to-head for new customers. Last, backbone 2 may fear to become the victim of another targeted degradation if the first one is not successful. For example, backbone 2's lower profitability when it offers transit may weaken its financial health to the point that a targeted degradation would provoke bankruptcy, thereby making this strategy more appealing for the dominant backbone.

To conclude, we do not wish to argue that targeted degradation is always profitable for the dominant backbone, but simply that such a backbone will find it is an appealing and robust strategy in a variety of circumstances.

\section{Multihoming}

Currently some of the largest ISPs "multihome", that is are customers of several backbones. Multihoming provides insurance both against the breakdown of connection to one's backbone and against the breakdown of one's

backbone 2's incentive to get out of the degradation region is necessarily larger than backbone 1's incentive to remain in it. 
backbone $^{51}$. For the same reasons, some of firms that are large users of the Internet multihome on several ISPs, which may be linked to different backbones. Multihoming is represented on figure 5, where Internet backbone provider 1 , the dominant backbone, has degraded its connection with the small IBP (this is represented by the bold vertical double line). The ISP is a customer of both backbones, and enjoys perfect connection with the clients of both IBPs. This section shows that, in the context of our model, the degradation of interconnection by a dominant backbone would in general be profitable even if customers multihome.

Multihoming might impair a dominance-enhancing degradation strategy in two ways. First, pre-existing multihoming by some installed base customers softens the impact of the degradation strategy on both the dominant and targeted networks. It makes it less costly for the dominant network to reduce the connectivity of its own customers with those of its rivals, and, as long as the difference in installed bases remains the same, it has no impact on the relative quality of the networks. Section 7.1 confirms this intuition and shows that, in general, pre-existing multihoming by customers in the installed base encourages degradation.

Second, customers, whether new or "singlehoming" members of the installed base, may choose to multihome in order to increase connectivity in the presence of degradation. Section 7.2 shows that the smaller backbones cannot use this fact in order to circumvent the degradation of the connection. In order to induce multihoming, the smaller backbone must expand output and this leads to a decrease in prices which, in many cases, will lead to a decrease in its profit. While we certainly do not wish to claim that incentives for multihoming are never increased by degradation, this analysis clearly shows that multihoming need not impair the profitability of the degradation strategy.

Before embarking in the analysis of these two points, we should note that we are overstating in three ways the consequences of multihoming. First, we assume that multihoming does not increase the costs of the customer, apart from the fact that it must pay both backbones. We thereby assume away one of the main impediments to its widespread use as a defense mechanism - it is technologically costly:

1. The use of the Border Gateway Protocol version 4 (BGP4), which must be run by ISPs or end users who multihome, requires very qualified staff and is prohibitively expensive for smaller clients. Multihoming

\footnotetext{
${ }^{51}$ The first but not the second type of insurance can also be obtained by having multiple links to the same backbone.
} 
may also impose routing costs on the backbones, which may charge the multihoming customer.

2. There are transaction costs associated with multiple relationships.

3. Connecting to multiple backbones implies a loss of returns to scale: because the cost of a connection is less than proportional to its bandwidth, splitting one's traffic among several backbones increases the total connection cost.

Second, by assuming that multihoming ensures perfect connectivity, even in the presence of complete degradation of the connection between the backbones, we overstate its effectiveness. In practice, the multihoming client does not control the routing of inbound (return) traffic, and therefore multihoming is only partly successful in alleviating degradation.

Third, if the dominant backbone perceived multihoming as a threat, it could impede its use by a number of techniques such as a refusal to deal with multihoming customers or to offer BGP4. These overt techniques could possibly trigger antitrust action, and so more subtle techniques such as high charges for the use of BGP4, volume discounts (which imply a greater loss of returns to scale), and the slowing down of connections to multihoming customers, ${ }^{52}$ would probably be more effective.

\subsection{Pre-existing multihoming}

In this subsection, we assume that, for reasons exogenous to the model, some users in the installed base already multihome before degradation takes place, and we explore the consequences of this fact on the incentives of the dominant backbone. We assume that the new customers and that the non already multihoming users in the installed base cannot multihome. This assumption is lifted in subsection 7.2.

\subsubsection{The duopoly case}

We use the framework of section 4, and we modify it by assuming that by part of the customers in the installed based multihome. More precisely, we assume that the installed base, still of size $\beta=\beta_{1}+\beta_{2}$, is divided into

- a fraction $\beta_{i} \Leftrightarrow \alpha / 2$ of single-homers attached to each backbone $i=1,2$ with, as above, $\beta_{1} \geq \beta_{2}$,

\footnotetext{
${ }^{52}$ In this respect, the dominant network could build a reputation for not being cooperative with the upgrading of connections to multihoming customers.
} 
- a fraction $\alpha$ of multi-homers attached to both backbones.

This implies that the total installed base has the same size $\beta=\beta_{1}+\beta_{2}$ as before and that the installed base advantage of backbone 1 is still $\Delta=\beta_{1} \Leftrightarrow \beta_{2}$. The number of new customers in the Cournot equilibrium is given by

$$
q_{i}^{*}(\alpha, \theta)=q_{i}^{*}(0, \theta)+\frac{\alpha}{2}\left(1 \Leftrightarrow \frac{3(1 \Leftrightarrow v)}{3 \Leftrightarrow v(2+\theta)}\right)
$$

where $q_{i}^{*}(0, \theta)$ is the no multihoming equilibrium quantity of equation (5). Because $3(1 \Leftrightarrow v) \leq 3 \Leftrightarrow v(2+\theta)$, for given $\theta$ the presence of multihoming increases the equilibrium quantities (strictly if $\theta<1$ ): indeed, multihoming makes both backbones more attractive to new customers and shifts the demand curve outwards.

We can prove that the results of section 4 extend to multihoming by the installed base:

Proposition 7 In the presence of multihoming by the installed base, the larger backbone prefers a lower quality of interconnection than the smaller backbone: $\theta_{1}^{*} \leq \theta_{2}^{*}$ with a strict inequality if $F(\theta)=0$ for all $\theta$ and $\theta_{2}^{*}>0$. Moreover, its preferred quality of interconnection decreases when its installed base advantage increases: keeping the total installed base $\beta$ constant, $\theta_{1}^{*}$ is a (weakly) decreasing function of $\Delta$.

The proof is similar ${ }^{53}$ to that of proposition 1.

We now turn to the impact of the extent of multihoming (measured by $\alpha$ ) on equilibrium. The difference in quality

$$
s_{1} \Leftrightarrow s_{2}=v(1 \Leftrightarrow \theta)\left[\beta_{1} \Leftrightarrow \beta_{2}+q_{1}^{*}(\alpha, \theta) \Leftrightarrow q_{2}^{*}(\alpha, \theta)\right]
$$

is independent of $\alpha$. Thus, multihoming has no impact on the quality differential created by degradation. In contrast, as we have already noted, multihoming reduces the impact on total demand of a decrease in connectivity. It is therefore not surprising that multihoming may encourage degradation:

\footnotetext{
${ }^{53}$ In order to show that the equilibrium $\theta$ is decrasing in $\Delta$, write $q_{1}^{*}(\alpha, \theta)=\psi(\theta)+$ $\alpha \phi(\theta)+\Delta \rho(\theta)$. Then

$$
\frac{1}{2} \frac{\partial^{2} q_{1}^{*}(\alpha, \theta)}{\partial \Delta \partial \theta}=(\psi(\theta) \rho(\theta))^{\prime}+\alpha(\phi(\theta) \rho(\theta))^{\prime}+\Delta\left(\rho(\theta)^{2}\right)^{\prime}
$$

The proof is then similar to that of footnote 35 , with the added statement that $\phi(\theta) \rho(\theta)$ is decreasing as both $\phi$ and $\rho$ are.
} 
Proposition 8 Under duopoly, if connectivity is costless $(F(\theta)=0$ ), the equilibrium quality of interconnection of the backbones decreases with the extent of installed base multihoming $\alpha$.

The proof ${ }^{54}$ relies on the fact that when $F(\theta)=0$ the quality that maximizes the profit of a backbone is also the quality that maximizes its equilibrium quantity. The analysis is more complex with costly connectivity, and we have been unable to generalize this proposition or to find a counterexample, although we have been able to show the partial result summarized in the following proposition.

Proposition 9 Under duopoly, with costly connectivity, there exits an $\bar{\alpha}$, maybe equal to 0 or 1 , such that the equilibrium quality of interconnection is equal to 1 for $\alpha \leq \bar{\alpha}$ and is smaller than 1 for $\alpha>\bar{\alpha}$.

Proof. To show the result, it is sufficient to prove that if $\theta_{1}^{*}$ is strictly smaller than 1 for some $\alpha$, it is also strictly smaller for all $\alpha^{\prime}>\alpha$. Therefore, assume that we have (with obvious notation)

$$
\pi_{1}^{*}(\alpha, \theta) \Leftrightarrow F(\theta) \geq \pi_{1}^{*}(\alpha, 1) \Leftrightarrow F(1) .
$$

Because $q_{1}^{*}$ and therefore $\pi_{1}^{*}$ are strictly increasing in $\alpha$ for $\theta<1$, we have $\pi_{1}^{*}\left(\alpha^{\prime}, \theta\right) \Leftrightarrow F(\theta)>\pi_{1}^{*}(\alpha, \theta) \Leftrightarrow F(\theta)$. Because $q_{1}^{*}$ and therefore $\pi_{1}^{*}$ do not depend on $\alpha$ when $\theta=1$, we have $\pi_{1}^{*}(\alpha, 1) \Leftrightarrow F(1)=\pi_{1}^{*}\left(\alpha^{\prime}, \theta=1\right) \Leftrightarrow F(1)$. Therefore

$$
\pi_{1}^{*}\left(\alpha^{\prime}, \theta\right) \Leftrightarrow F(\theta)>\pi_{1}^{*}(\alpha, 1) \Leftrightarrow F(1),
$$

which proves the result.

In exactly the same way, we can prove the following proposition:

Proposition 10 It $\theta$ only takes values 0 and 1, then under duopoly the equilibrium quality of connection is decreasing in $\alpha$.

\subsubsection{Targeted degradation}

Turning to the framework of section 6 , we assume that the installed base, whose total size is still $\beta$, is divided into:

- $(\beta \Leftrightarrow \alpha) / 2=\beta / 2 \Leftrightarrow \alpha / 2$ single-homers attached to the dominant backbone (backbone 1),

\footnotetext{
${ }^{54}$ It is a straightforward revealed preference, based on the fact that $\partial^{2} q_{i}^{*} / \partial \alpha \partial \theta$ is negative. This proves that the $\theta$ that maximizes $q_{i}^{*}$ decreases with $\alpha$. When $F(\theta)=0$, this is sufficient to prove the result. When $F(\theta)$ is not constant, a standard revealed preference argument would require us to first prove $\partial^{2}\left(q_{i}^{*}\right)^{2} / \partial \alpha \partial \theta<0$, which is not always true.
} 
- $(\beta \Leftrightarrow 2 \alpha) / 4=\beta / 4 \Leftrightarrow \alpha / 2$ single-homers attached to the targeted backbone (backbone 3 ),

- $\alpha$ multi-homers attached to both the dominant and the targeted backbones (backbones 1 and 3),

- $\frac{\beta}{4}$ of single-homers attached to the non-targeted small backbone (backbone 2),

with $\alpha<\beta / 2$. We could assume that there is some multihoming between backbone 2 and backbones 1 and 3 , but it would be irrelevant as long as backbone 2 keeps perfect connectivity with the other two backbones.

It is straightforward to show that if the dominant backbone adopts the accommodation strategy $^{55}\left(\theta_{i j}=1\right)$, then the other two follow suit. The profit function of each backbone $i$ is the same as in section 6.3 and the equilibrium is the same as above: the difference between the installed bases is irrelevant to the new customers.

If backbone 1 opts for the global degradation strategy $\left(\theta_{12}=\theta_{13}=0\right)$, one can again prove that backbones 2 and 3 find it optimal to interconnect $\left(\theta_{23}=\right.$ 1 ), and that, as in section 6 , the dominant network finds accommodation preferable to global degradation.

Consider now the strategy of targeted degradation, where, as above, backbone 1 does not interconnect with backbone $3\left(\theta_{13}=0\right)$, maintains a high quality of interface with backbone $2\left(\theta_{12}=1\right)$ as long as this backbone does not offer transit to backbone 3 , and limits the capacity of the interface with backbone 2 .

As in section 6 , we only consider the case where backbone 3 attracts no new customers. ${ }^{56}$ Then, the equilibrium is:

$$
\begin{aligned}
\hat{q}_{1}^{t}(\alpha) & =\frac{1}{6} \frac{2(1 \Leftrightarrow c)+v \beta+2 v \alpha}{1 \Leftrightarrow v}, \\
\hat{q}_{2}^{t}(\alpha) & =\frac{1}{12} \frac{4(1 \Leftrightarrow c)+5 v \beta \Leftrightarrow 2 v \alpha}{1 \Leftrightarrow v},
\end{aligned}
$$

\footnotetext{
${ }^{55} \mathrm{As}$ in section 6 , we assume that the quality of connection (the $\theta_{i j} \mathrm{~s}$ ) can only take values 0 and 1 , and $F(1)=F(0)=0$.

${ }^{56}$ When backbone 3 attracts new customers, backbone 1 benefits from targeted degradation when its installed base is large enough and there if a large amount of multihoming, as long as backbone 2 does not offer transit to the targeted backbone, which is always the case if $v \leq 1 / 3$ (if $v>1 / 3$, one may needs to put an upper bound on $\alpha$ and/or $\beta$ to rule out transit).
} 
and $\hat{\pi}_{i}^{t}(\alpha)=(1 \Leftrightarrow v)\left(\hat{q}_{i}^{t}(\alpha)\right)^{2}$. Since the equilibrium profit of backbone 1 under targeted degradation increases with $\alpha$ while the accommodation equilibrium profit is independent of $\alpha$, the targeted degradation strategy is even more desirable than above. ${ }^{57}$ The intuition is straightforward. With multihoming $s_{2} \Leftrightarrow s_{1}$ decreases, as the new customers who choose backbone 1 benefit from connectivity with some of the customers of network 3. Hence, the quality differentiation advantage of network 2 is decreased. Furthermore, and as in the duopoly case, the contraction in demand caused by degradation is lowered by pre-existing multihoming, since backbone 1 maintains connectivity with part of the installed base of backbone 3. Both effects make targeted degradation more attractive when part of the installed base already multihomes.

Proposition 11 Assume $v>1 / 3$ and $\beta \in(1,4(1 \Leftrightarrow 2 v) /(1+v))$ and that $\alpha$ is "small". Then,

i. targeted degradation prevents the target from attracting any new customer;

ii. and pre-existing multihoming raises the profitability of targeted degradation by the dominant backbone by

(a) reducing the demand contraction caused by degradation

(b) and by reducing the quality advantage enjoyed by the smaller nontargeted backbone.

From equation (10) it is straightforward to prove the following corollary:

Proposition 12 Assume $v>1 / 3$ and $\beta \in(1,4(1 \Leftrightarrow 2 v) /(1+v))$ and that for $i=2,3$, a small number $\alpha_{i}$ of consumers multihome between backbone $i$ and backbone 1. Assume further that any targeted backbone is unable to attract any new customer. Then, backbone 1 will target the backbone $i$ with the largest $\alpha_{i}$.

\subsection{Multihoming in response to degradation}

In this subsection, we show that the smaller backbone cannot in general defend itself against a degradation of connectivity by using the fact that new customers and/or the non already multihoming customers of the installed base could choose to multihome.

\footnotetext{
${ }^{57}$ Indeed, letting the superscript $a$ indicate accomodation, assume $\hat{\pi}_{1}^{t}(0) \geq \hat{\pi}_{1}^{a}(0)$, we have $\hat{\pi}_{1}^{t}(\alpha)>\hat{\pi}_{1}^{t}(0) \geq \hat{\pi}_{1}^{a}(0)=\hat{\pi}_{1}^{a}(1)$, which proves the result.
} 


\subsubsection{Duopoly}

We return to the framework of section 4, assuming for simplicity that there is no pre-existing multihoming. Furthermore, we consider only cases where degradation, if it occurs is extreme, so that $\theta$ can only take values 0 or 1 . When $\theta=1$, the customers have no incentive to multihome and the equilibrium identified in section 4 is still an equilibrium.

To study the consequences of a degradation of connectivity $(\theta=0)$, we need to introduce some new notation. Let

- $\eta_{i}$ be the number of customers in the installed base of backbone $j \neq i$ who multihome on backbone $i$;

- $\eta$ be the number of new customers who multihome;

- $q_{i}$ be the number of new customers who contract only with backbone $i$.

Then,

$$
s_{i}=v\left[\left(q_{i}+\eta\right)+\left(\beta_{i}+\eta_{i}\right)\right]
$$

is the quality of service for customers who singlehome with backbone $i$, and

$$
s=v\left[q_{1}+q_{2}+\eta+\beta_{1}+\beta_{2}\right]=s_{1}+s_{2} \Leftrightarrow v\left(\eta_{1}+\eta_{2}+\eta\right)
$$

is the quality of service for customers who multihome. Notice that $s$ is the sum of the qualities of backbones 1 and 2 corrected for double counting of connections: therefore it is smaller than or equal to $s_{1}+s_{2}$, and strictly smaller $\left(s<s_{1}+s_{2}\right)$ when there is multihoming.

The strategic variable selected by backbone $i$ is the number of new connections it offers

$$
Q_{i}=q_{i}+\eta_{i}+\eta
$$

In order to check whether the equilibrium quantities of equation (5) are still equilibrium quantities when multihoming is possible, we need to show that if the number of new connections offered by backbone 1 is fixed at $Q_{1}=q_{1}^{*}$, then backbone 2 has no incentive to offer more than $Q_{2}=q_{2}^{*}$ connections, even when customers have the possibility to multihome.

When there are at the same time multihoming and singlehoming customers, the quality adjusted prices must be the same for the three possibilities $^{58}$ open to consumers: connecting with backbone 1 , connecting with

\footnotetext{
${ }^{58} \mathrm{We}$ are assuming that both backbones attract new customers. The results still hold in backbone 2 if excluded from the market.
} 
backbone 2 or multihoming. This has two important consequences. First, it is impossible for some new customers to be unattached while others multihome: the marginal benefit of a first connection to backbone 2 is $\tau+v s_{2} \Leftrightarrow p_{2}$ whereas the marginal benefit of a second connection to backbone 2 is equal to $v\left(s \Leftrightarrow s_{1}\right) \Leftrightarrow p_{2}$, and therefore smaller (since $\left.s<s_{1}+s_{2}\right) .{ }^{59}$ Therefore, if there is any multihoming, every customer subscribes to at least one network:

$$
q_{1}+q_{2}+\eta=1
$$

Second, the price charged by backbone 2 is equal to the value of connecting to those customers reachable solely through backbone $2: p_{2}=s \Leftrightarrow s_{1}$. As we have seen in the preceding paragraph, all potential customers, and there are $1+\beta_{1}+\beta_{2}$ of them, are connected. If the number of new connections offered by 1 is fixed at $Q_{1}=q_{1}^{*}$, the price for backbone 2's services is given by

$$
p_{2}=v\left(s \Leftrightarrow s_{1}\right)=v\left[\left(1+\beta_{1}+\beta_{2}\right) \Leftrightarrow\left(\beta_{1}+q_{1}^{*}\right)\right]=v\left(1+\beta_{2} \Leftrightarrow q_{1}^{*}\right),
$$

and is thus independent from the number of connections offered by backbone 2 (provided that multihoming occurs, i.e., $Q_{2}>1 \Leftrightarrow q_{1}^{*}$ ).

Therefore, backbone 2 might as well offer the maximum possible number of connections, that is $1+\beta_{1}$, and a necessary and sufficient condition for network 2 not to induce multihoming, and therefore for $\left(q_{1}^{*}, q_{2}^{*}\right)$ to be an equilibrium is

$$
(1 \Leftrightarrow v)\left(q_{2}^{*}\right)^{2} \geq\left(v\left(\left(1 \Leftrightarrow q_{1}^{*}\right)+\beta_{2}\right) \Leftrightarrow c\right)\left(1+\beta_{1}\right) .
$$

A sufficient, but not necessary, condition for (13) to be satisfied is

$$
v\left[\left(1 \Leftrightarrow q_{1}^{*}\right)+\beta_{2}\right] \leq c,
$$

which is, for instance, satisfied if $\Delta$ is sufficiently large. ${ }^{60}$

\footnotetext{
${ }^{59}$ Formally,

$$
\hat{p}=p_{1}+p_{2}-s>p_{1}+p_{2}-s_{1}-s_{2}=2 \hat{p}
$$

implies $\hat{p}=0$ and therefore

$$
q_{1}+q_{2}+\eta=1 .
$$

${ }^{60}$ This condition can be shown to be equivalent to $q_{2}^{*}<1-\left(q_{1}^{*}+q_{2}^{*}\right)$, and an increase in $\Delta$ reduces $q_{2}^{*}$ by has no impact on $\left(q_{1}^{*}+q_{2}^{*}\right)$. In particular, $\left(q_{1}^{*}, q_{2}^{*}\right)$ is an equilibrium whenever $q_{2}^{*}=0$.
} 
Note finally that condition (13) is too stringent if only a fraction of the customers have the technological know-how to, say, master BGP4. If this were the case, backbone 2's margin, $\bar{p}_{2} \Leftrightarrow c$, in the multihoming region is still the same but the potential market for backbone 2 is then smaller than $\left(1+\beta_{1}\right)$.

Proposition 13 Under duopoly, the possibility of multihoming in response to degradation does not perturb the equilibrium if condition (13) holds.

Proposition 13 understates the extent of multihoming when it is both costless and allowed by the dominant backbone. First, we have assumed that the consumers' willingness to pay for access is positive $(\tau \in[0,1])$. However, a consumer with a negative ${ }^{61}$ willingness to pay for access might subscribe at a strictly positive price once the network externality is accounted for. Therefore, the proposition, as it stands, relies on a distributional assumption.

Second, we have assumed that all consumers have the same valuation of connectivity. In practice some consumers value connectivity more than others and even in a non-competitive industry, backbones would not perfectly discriminate on the basis of the consumers' willingness to pay for connectivity. To see this, suppose that within each installed base, a "very small" (so that their presence does not affect the above analysis of the equilibrium) fraction of customers have a higher valuation for connectivity, given by $(1+\rho) v$ with $\rho>0$. Intuitively, for $\rho$ large enough, those customers will want to "multihome", that is, to subscribe to the rival's backbone as well, even though they are already part of one backbone' s installed base. However, the customers who initially belong to the smaller backbone's installed base will be the most eager to multihome, and, as $\rho$ increases from 0 , those customers will be the first ones to be willing to multihome. More precisely, they will decide to "multihome" on backbone 1 as soon as

$$
\hat{s}_{1} \equiv(1+\rho) v\left(\beta_{1}+q_{1}\right) \geq p_{1}=\hat{p}+v\left(\beta_{1}+q_{1}\right),
$$

that is, when

$$
\rho \geq \rho_{2}=\frac{\hat{p}}{v\left(\beta_{1}+q_{1}\right)} .
$$

Similarly, the customers that initially belong to the larger installed base will be willing to multihome only when

$$
\rho \geq \rho_{1}=\frac{\hat{p}}{v\left(\beta_{2}+q_{2}\right)}>\rho_{2} .
$$

\footnotetext{
${ }^{61}$ This does not necessarily imply that such consumers positively do not want to be connected, but rather that the private costs of connection (for instance, buying a computer and learning how to use it) are larger than the benefits that they receive from subscribing to the network.
} 
Proposition 14 Add to the basic model a small proportion of customers with a higher valuation for connectivity $((1+\rho) v$ instead of $v)$. There exists an interval $\left(\rho_{2}, \rho_{1}\right)$ such that for any $\rho$ in that interval, in the presence of degradation, these consumers

$i$. select the dominant backbone if unattached;

ii. multihome to the dominant backbone if they are part of the smaller backbone's installed base;

iii. do not multihome to the smaller backbone if they are part of the dominant backbone's installed base.

(If $\rho<\rho_{2}$, no multihoming occurs. If $\rho>\rho_{1}$, all the consumers with a high valuation for connectivity multihome.)

\subsubsection{Targeted degradation}

We now turn to our model of targeted degradation, and show that, in this case also, there is no multihoming at equilibrium. We keep the same hypotheses as in section 6: connectivity is either perfect $\left(\theta_{i j}=0\right)$ or totally degraded $\left(\theta_{i j}=\right.$ $0)$. Furthermore, we only consider cases where under targeted degradation backbone 3 does not sell to any new customer.

If the dominant backbone pursues a strategy of global accommodation, multihoming can bring no connectivity benefit, and the equilibrium of section 6 is still an equilibrium. We now show that this conclusion is also valid with targeted degradation.

In the non multihoming equilibrium, the benefit that the marginal customer would receive from signing onto network 3 must be less that $c$, otherwise network 3 could expand its capacity by a small amount, attract one customer and increase its profit. Therefore

$$
1 \Leftrightarrow q_{1}^{*} \Leftrightarrow q_{2}^{*}+v\left(\frac{\beta}{2}+q_{2}^{*}\right) \leq c .
$$

With multihoming, the price that backbone 3 would receive would be $s \Leftrightarrow s_{1}$, the value of the extra service it provides, where

$$
s=s_{2}=v\left(q_{1}^{*}+q_{2}^{*}+q_{3}+\beta\right)=v(1+\beta)
$$

is the quality of universal connection. Because $s_{1}=v\left(q_{1}^{*}+q_{2}^{*}+3 \beta / 4\right)$,

$$
\begin{aligned}
p_{3} & =v\left(1+\frac{\beta}{4} \Leftrightarrow q_{1}^{*} \Leftrightarrow q_{2}^{*}\right) \\
& \leq c \Leftrightarrow v\left(\frac{\beta}{4}\right) \Leftrightarrow v q_{2}^{*}<c,
\end{aligned}
$$


and backbone 3 has no incentives to expand to induce multihoming.

Proposition 15 The possibility of multihoming in response to degradation of connectivity does not change the parameter values for which a targeted degradation equilibrium arises. 


\section{References}

European Commission (1998), Commission decision of 8 july 1998 declaring a concentration to be compatible with the common market and the functionning of the EEA agreement (case IV/M.1069 - WorldCom/MCI). Official Journal of the European Commission, 4.5.1999, L116/1-L116/35.

Farrell, J. \& G. Saloner (1985), 'Standardization, compatibility and innovation', Rand Journal of Economics 16, 70-83.

Fudenberg, Drew \& Jean Tirole (1997), Poaching. mimeo, IDEI and Harvard University.

Gabszewicz, J. \& J.-F. Thisse (1979), 'Price competition, quality and income disparities', Journal of Economic Theory 22, 327-338.

Gupta, A., D. Stahl \& A. Whinston (1994), Managing the internet as an economic system. mimeo, University of Texas, Austin.

Hansmann, Henry (1996), The Ownership of Enterprise, Harvard University Press, Cambridge.

Innes, R \& R. Sexton (1993), 'Customer coalitions, monopoly price discrimination and generic entry deterrence', European Economic Review 37, 1569-1597.

Journal of Industrial Economics Symposium on Compatibility (1992), in Journal of Industrial Econornics, XL(1).

Katz, M. \& C. Shapiro (1985), 'Network externalities, competition, and compatibility', American Economic Review 75, 424-440.

MacKie-Mason, J. \& H. Varian (1995a), 'Pricing congestible network resources', IEEE Journal of Selected Areas in Communication 13, 11411149 .

MacKie-Mason, J. \& H. Varian (1995b), Pricing the internet, in B.Kahin \& J.Keller, eds, 'Public Access to the Internet', Prentice Hall, Englewood Cliffs.

Marcus, Scott (forthcoming), How to Design Wide Area Internetworks, Addison Wesley, Longman. 
Richard, J. (1999), 'Backbone performance measurement'.

URL: http://www.boardwatch.internet.com/isp/spring99/measure.html

Rohlfs (1974), 'A theory of interdependent demand for a communications service', Bell Journal of Economics 5, 16-37.

Salant, S. W., S. Switzer \& R. J. Reynolds (1983), 'Losses from horizontal mergers: The effects of an exogeneous change in industry structure on cournot-nash equilibrium', Quarterly Journal of Economics 48, 185-199.

Shaked, A. \& J. Sutton (1982), 'Relaxing price competition through product differentiation', Review of Economic Studies 49, 3-13.

Shenker, S., D. Clark, D. Estrin \& S. Herzog (1996), 'Pricing in computer networks: Reshaping the research agenda', Telecommunications Policy 20, 183-201.

Tirole, Jean (1988), The Theory of Industrial Organization, MIT Press, Cambridge. 


\section{Appendix}

\section{A Proof of Proposition 2}

The profit function $\pi_{i}^{*}=(1 \Leftrightarrow v)\left(q_{i}^{*}\right)^{2}$ is convex in $\theta$, since $q_{i}^{*}$ is nonnegative and convex. ${ }^{62}$ Therefore, if maintaining connectivity is costless, the only relevant choices for backbone 1 are $\theta=0$ and $\theta=1$. The former is optimal if $q_{1}^{*}$ is higher for $\theta=0$ than for $\theta=1$, that is, if

$$
\frac{2(1 \Leftrightarrow c)+v \beta}{3 \Leftrightarrow 2 v}+\frac{v \Delta}{1 \Leftrightarrow 2 v}>\frac{2(1 \Leftrightarrow c)+2 v \beta}{3(1 \Leftrightarrow v)},
$$

or

$$
\begin{aligned}
\Delta & >\frac{1 \Leftrightarrow 2 v}{v}\left[\frac{2(1 \Leftrightarrow c)+2 v \beta}{3(1 \Leftrightarrow v)} \Leftrightarrow \frac{2(1 \Leftrightarrow c)+v \beta}{3 \Leftrightarrow 2 v}\right] \\
& =\frac{1 \Leftrightarrow 2 v}{3(1 \Leftrightarrow v)(3 \Leftrightarrow 2 v)}[2(1 \Leftrightarrow c)+(3 \Leftrightarrow v) \beta]=\Delta^{*} .
\end{aligned}
$$

Last, it can be checked that the functions $\frac{1-2 v}{(1-v)(3-2 v)}$ and $\frac{(3-v)(1-2 v)}{(1-v)(3-2 v)}$ are both decreasing in $v$ over the range $[0,1 / 2]$, so that the threshold $\Delta^{*}$ decreases with $v$.

\section{B Proof of Proposition 3}

Note that the equilibrium gross profits are given by $\pi_{i}^{*}=(1 \Leftrightarrow v)\left(q_{i}^{*}\right)^{2}$ where $q_{i}^{*}$ is of the form $\psi(\theta)+\rho(\theta) \Delta$, with both $\rho$ and $\psi \rho$ nonnegative and nonincreasing in $\theta$.

i) Proof that $\theta_{1}^{*} \leq \theta^{*} \leq \theta_{2}^{*}$ : Define $G(\theta, \lambda)=\lambda\left(\pi_{1}^{*} \Leftrightarrow F\right)+(1 \Leftrightarrow \lambda)\left(\pi_{2}^{*} \Leftrightarrow F\right)$. We have:

\footnotetext{
${ }^{62} q_{i}^{*}$ is of the form $\psi(\theta)+\rho(\theta) \Delta_{i}$, where both $\psi$ and $\rho$ are convex in $\theta$.
} 


$$
\begin{aligned}
\frac{\partial^{2} G}{\partial \theta \partial \lambda} & =\frac{\partial^{2}\left[\lambda\left(\pi_{1}^{*} \Leftrightarrow F\right)+(1 \Leftrightarrow \lambda)\left(\pi_{2}^{*} \Leftrightarrow F\right)\right]}{\partial \theta \partial \lambda} \\
& =\frac{\partial\left[\pi_{1}^{*} \Leftrightarrow \pi_{2}^{*}\right]}{\partial \theta} \\
& =(1 \Leftrightarrow v) \frac{\partial\left[(\psi(\theta)+\rho(\theta) \Delta)^{2} \Leftrightarrow(\psi(\theta) \Leftrightarrow \rho(\theta) \Delta)^{2}\right]}{\partial \theta} \\
& =(1 \Leftrightarrow v) \frac{\partial[4 \psi(\theta) \rho(\theta) \Delta]}{\partial \theta} \\
& \leq 0
\end{aligned}
$$

since $\psi \rho$ is a nonincreasing function of $\theta$. A revealed preference argument then establishes that $\theta(\lambda)=\arg \max _{\theta} G(\theta, \lambda)$ (weakly) increases with $\lambda$, and thus (applying this to $\lambda=0,1 / 2$ and 1 ), $\theta_{1}^{*} \leq \theta^{*} \leq \theta_{2}^{*}$.

ii) Proof that $\partial \theta^{*} / \partial \Delta \leq 0$ : Similarly:

$$
\begin{aligned}
\frac{\partial^{2}\left[\pi_{1}^{*}+\pi_{2}^{*} \Leftrightarrow 2 F\right]}{\partial \theta \partial \Delta} & =\frac{\partial^{2}\left[(\psi(\theta)+\rho(\theta) \Delta)^{2}+(\psi(\theta) \Leftrightarrow \rho(\theta) \Delta)^{2} \Leftrightarrow 2 F\right]}{\partial \theta \partial \Delta} \\
& =2 \frac{\partial^{2}\left[\psi^{2}(\theta)+\rho^{2}(\theta) \Delta^{2}\right]}{\partial \theta \partial \Delta} \\
& =4 \frac{\partial\left[\rho^{2}(\theta) \Delta\right]}{\partial \theta} \\
& \leq 0,
\end{aligned}
$$

where the last inequality stems from the fact that $\rho$ is nonnegative and nonincreasing in $\theta$. The conclusion then follows again from a standard revealed preference argument.

\section{Proof of Proposition 4}

Backbone $i$ 's profit is

$$
\pi_{i}=\left[1 \Leftrightarrow q_{i} \Leftrightarrow \sum_{j \neq i} q_{j}+v\left(\frac{\beta}{4}+q_{i}+\sum_{j \neq i} \theta_{i j}\left(\frac{\beta}{4}+q_{j}\right)\right) \Leftrightarrow c\right] q_{i},
$$

and its best response thus satisfies

$$
2(1 \Leftrightarrow v) q_{i}+\sum_{j \neq i}\left(1 \Leftrightarrow \theta_{i j} v\right) q_{j}=1 \Leftrightarrow c+\left(1+\sum_{j \neq i} \theta_{i j}\right) \frac{v \beta}{4},
$$


while equilibrium profits from new customers are as before directly related to the equilibrium quantities $\left(\pi_{i}^{*}=(1 \Leftrightarrow v)\left(q_{i}^{*}\right)^{2}\right)$.

Degrading the connectivity between $i$ and $k$, say, decreases the demand for both $i$ and $k$ without creating any advantage for one over the other, and furthermore creates an advantage for their rivals. This is reflected in conditions $(A .1)$, which show that decreasing $\theta_{i k}$ adversely affects the response functions of backbones $i$ and $k$ in the same way and does not affect the other backbones' responses; as a result, backbone $i$ 's equilibrium capacity and profit decreases. If for example all connectivity levels but $\theta_{i k}$ are perfect, backbone $i$ 's equilibrium capacity, as a function of $\theta_{i k}$, is given by

$$
q_{i}^{*}=\frac{1 \Leftrightarrow c+\left(1+3 \theta_{i k}\right) \frac{v \beta}{4}}{5(1 \Leftrightarrow v)+3\left(1 \Leftrightarrow \theta_{i k} v\right)},
$$

and increases with $\theta_{i k}$. By inspection, it can be checked that all other degradation strategies are non profitable as well. Therefore, in equilibrium, all $\theta_{i j}$ are equal to 1 , and the equilibrium profits and capacities are the same for all four backbones.

\section{Proof of Proposition 5}

The proof follows the same line as for the previous proposition and we only sketch it here. Backbone $i$ 's profit is (for $i \neq j=2,3$ )

$$
\pi_{i}=\left[1 \Leftrightarrow q_{i} \Leftrightarrow q_{1} \Leftrightarrow q_{j}+v\left(\frac{\beta}{4}+q_{i}+\theta_{1 i}\left(\frac{\beta}{2}+q_{1}\right)+\theta_{i j}\left(\frac{\beta}{4}+q_{j}\right)\right) \Leftrightarrow c\right] q_{i},
$$

whereas the dominant backbone's profit is

$$
\pi_{1}=\left[1 \Leftrightarrow q_{1} \Leftrightarrow q_{2} \Leftrightarrow q_{3}+v\left(\frac{\beta}{2}+q_{1}+\theta_{12}\left(\frac{\beta}{4}+q_{2}\right)+\theta_{13}\left(\frac{\beta}{4}+q_{3}\right)\right) \Leftrightarrow c\right] q_{1} .
$$

Best responses thus satisfy

$$
\begin{aligned}
& 2(1 \Leftrightarrow v) q_{1}+\left(1 \Leftrightarrow \theta_{12} v\right) q_{2}+\left(1 \Leftrightarrow \theta_{13} v\right) q_{3}=1 \Leftrightarrow c+\frac{v \beta}{2}+\left(\theta_{12}+\theta_{13}\right) \frac{v \beta}{4}, \\
& 2(1 \Leftrightarrow v) q_{i}+\left(1 \Leftrightarrow \theta_{12} v\right) q_{1}+\left(1 \Leftrightarrow \theta_{23} v\right) q_{j}=1 \Leftrightarrow c+\theta_{12} \frac{v \beta}{2}+\left(1+\theta_{23}\right) \frac{v \beta}{4},
\end{aligned}
$$

and, again, $\pi_{i}^{*}=(1 \Leftrightarrow v)\left(q_{i}^{*}\right)^{2}$. 
i. Degrading the connectivity between the two small backbones $\left(\theta_{23}=0\right)$ adversely affects their response functions, does not affect the dominant backbone's behavior and is not profitable for them. Degrading the connectivity between, say, backbones 1 and 2, affects the reaction function of backbone 2 more than the reaction function of backbone 1 (and does not affect the other small backbone's response function), and is thus even less profitable for backbone 2 .

ii. Degrading the two connections with the small backbones adversely affects all response functions. If $\theta_{23}=1$ and $\theta_{12}=\theta_{13}=0$, the dominant backbone's equilibrium capacity is then given by (assuming that $v \leq 1 / 3$, otherwise tipping would occur, yielding $q_{1}=0$ ):

$$
q_{1}^{g}=\frac{(1 \Leftrightarrow 3 v)\left(1 \Leftrightarrow c+\frac{v \beta}{2}\right)}{2\left(2 \Leftrightarrow 6 v+3 v^{2}\right)},
$$

where the superscript $g$ stand for "global degradation" while if $\theta_{12}=$ $\theta_{13}=\theta_{23}=1$, it is given by (with the superscript $a$ standing for "accommodation")

$$
q_{1}^{a}=\frac{1 \Leftrightarrow c+v \beta}{4(1 \Leftrightarrow v)} .
$$

It is straightforward to check that $q_{1}^{g}<q_{1}^{a}$, and thus that the dominant backbone prefers to maintain connectivity.

\section{E Proof of Proposition 6}

Consider now the strategy of targeted degradation, where backbone 1 does not interconnect with backbone $3\left(\theta_{13}=0\right)$ but maintains a high quality of interface with backbone $2\left(\theta_{12}=1\right)$ as long as this backbone does not offer transit to backbone 3 .

Offering transit would amount for backbone 2 to revert to same situation as with global degradation $\left(\theta_{23}=1\right.$ but $\left.\theta_{12}=\theta_{13}=0\right)$. If instead backbone 2 does not offer transit services, then $\theta_{12}=\theta_{23}=1$ and $\theta_{13}=0$ and the profits are given by:

$$
\begin{aligned}
& \pi_{1}=\left[1 \Leftrightarrow q_{1} \Leftrightarrow q_{2} \Leftrightarrow q_{3}+v\left(\frac{3 \beta}{4}+q_{1}+q_{2}\right) \Leftrightarrow c\right] q_{1}, \\
& \pi_{2}=\left[1 \Leftrightarrow q_{1} \Leftrightarrow q_{2} \Leftrightarrow q_{3}+v\left(\beta+q_{1}+q_{2}+q_{3}\right) \Leftrightarrow c\right] q_{2}, \\
& \pi_{3}=\left[1 \Leftrightarrow q_{1} \Leftrightarrow q_{2} \Leftrightarrow q_{3}+v\left(\frac{\beta}{2}+q_{2}+q_{3}\right) \Leftrightarrow c\right] q_{3} .
\end{aligned}
$$


Since it is perfectly connected, backbone 2 now enjoys both a larger demand a quality advantage over the other two backbones; as a result, it can be checked that its equilibrium capacity and profit is higher than in the previous case (global degradation). Backbone 2 thus chooses not to offer transit.

Backbone 3 is the least well connected, as it has no access to the dominant backbone's installed base. As a result, its equilibrium capacity and profit are the lowest among the three backbones. It may even be unable to attract any new customer (i.e. $q_{3}=0$ in equilibrium); straightforward computations show that this is the case when

$$
\frac{v \beta}{4}>\frac{(1 \Leftrightarrow 2 v)(1 \Leftrightarrow c)}{1+v} .
$$

Then, letting the superscript $t$ stand for "targeted degradation" the dominant backbone's equilibrium capacity is

$$
\begin{aligned}
& \hat{q}_{1}^{t}=\frac{1}{6} \frac{2(1 \Leftrightarrow c)+v \beta}{1 \Leftrightarrow v}, \\
& \hat{q}_{2}^{t}=\frac{1}{12} \frac{4(1 \Leftrightarrow c)+5 v \beta}{1 \Leftrightarrow v},
\end{aligned}
$$

and as before $\hat{\pi}_{1}^{t}=(1 \Leftrightarrow v)\left(\hat{q}_{1}^{t}\right)^{2}$. Backbone 1 thus prefers the targeted degradation strategy to accommodation if $q_{1}^{t}>q_{1}^{a}$, which is equivalent to

$$
v \beta<1 \Leftrightarrow c .
$$

This inequality is compatible with (A. 2) if

$$
\frac{4(1 \Leftrightarrow 2 v)}{1+v}(1 \Leftrightarrow c)<v \beta<1 \Leftrightarrow c,
$$

which is the case if

$$
v>\frac{1}{3}
$$

Therefore, if $v>1 / 3$ and $c<1 \Leftrightarrow v \beta(\Leftrightarrow v \beta<1 \Leftrightarrow c)$, the targeted degradation strategy is profitable for the dominant backbone whenever it removes the targeted backbone as an active competitor.

\section{F Transit decision under myopic behavior}

Consider the above targeted degradation strategy and assume that it removes backbone 3 as a competitor. Then, after the first period, the remaining backbones' installed bases are, respectively: 


$$
\begin{aligned}
& \hat{\beta}_{1}=\beta_{1}+\hat{q}_{1}=\frac{\beta}{2}+\frac{1}{6} \frac{2(1 \Leftrightarrow c)+v \beta}{1 \Leftrightarrow v}, \\
& \hat{\beta}_{2}=\beta_{2}+\hat{q}_{2}=\frac{\beta}{4}+\frac{1}{12} \frac{4(1 \Leftrightarrow c)+5 v \beta}{1 \Leftrightarrow v} .
\end{aligned}
$$

The total installed base at date 2 is

$$
\hat{\beta}=\hat{\beta}_{1}+\hat{\beta}_{2}=\frac{3 \beta}{4}+\frac{1}{12} \frac{8(1 \Leftrightarrow c)+7 v \beta}{1 \Leftrightarrow v},
$$

while the difference in installed base is

$$
\hat{\Delta}=\hat{\beta}_{1} \Leftrightarrow \hat{\beta}_{2}=\frac{\beta}{4} \Leftrightarrow \frac{3}{12} \frac{v \beta}{1 \Leftrightarrow v}=\frac{1 \Leftrightarrow 2 v}{1 \Leftrightarrow v} \frac{\beta}{4} .
$$

The dominant backbone does not want to degrade connectivity in the second period if

$$
\hat{\Delta}<\Delta^{*}(\hat{\beta})=\frac{1 \Leftrightarrow 2 v}{3(1 \Leftrightarrow v)(3 \Leftrightarrow 2 v)}[2(1 \Leftrightarrow c)+(3 \Leftrightarrow v) \hat{\beta}] .
$$

It can be shown that the stronger condition $\hat{\Delta}<\Delta^{*}(3 \beta / 4)$ is satisfied (that is, the dominant backbone has no incentive to degrade connectivity even if we ignore the expansion in the total installed base). This condition writes as

$$
\frac{1 \Leftrightarrow 2 v}{1 \Leftrightarrow v} \frac{\beta}{4}<\frac{1 \Leftrightarrow 2 v}{3(1 \Leftrightarrow v)(3 \Leftrightarrow 2 v)}\left[2(1 \Leftrightarrow c)+(3 \Leftrightarrow v) \frac{3 \beta}{4}\right]
$$

and is satisfied if it is so when $c=1$, in which case it boils down to

$$
\frac{\beta}{4}<\frac{(3 \Leftrightarrow v)}{(3 \Leftrightarrow 2 v)} \frac{\beta}{4}
$$

which is always true whenever there are network externalities $(v>0)$. 